\title{
Wave breaking in the surf zone and deep water in a non-hydrostatic RANS model. Part 2: Turbulence and mean circulation
}

\author{
Morteza Derakhti ${ }^{1}$, James T. Kirby ${ }^{1}$, Fengyan $\mathrm{Shi}^{1}$ and Gangfeng $\mathrm{Ma}^{2}$ \\ ${ }^{1}$ Center for Applied Coastal Research, University of Delaware, Newark, DE, USA \\ ${ }^{2}$ Department of Civil and Environmental Engineering, Old Dominion University, \\ Norfolk, VA, USA \\ Corresponding author: Email address: derakhti@udel.edu
}

\begin{abstract}
Field-scale modeling of wave-breaking-induced turbulence and mean circulation is still challenging. Although Boussinesq-type models have been successfully used to study field-scale wave transformation and breaking-driven circulation, they cannot provide turbulence or the vertical structure of velocity field. In addition, the applicability of such models is limited to shallow water. In Part 1 (Derakhti et al., 2016b) of this study, we showed that the non-hydrostatic $\sigma$-coordinate RANS model NHWAVE, as described by Derakhti et al. (2016a), accurately predicts organized wave motions and total wave-breaking-induced energy dissipation from deep water up to the swash zone using a few vertical $\sigma$-layers. In this paper, our goal is to examine what level of detail of wave-breaking-induced turbulence and mean circulation, both in depth- and steepness-limited breaking waves, can be reproduced by NHWAVE. Further, effects of modeled turbulent eddy viscosity on the pre-
\end{abstract}


dicted time-averaged velocity distribution is discussed. We establish that NHWAVE is capable of predicting the structure of the mean velocity and vorticity fields including large-scale breaking-induced coherent vortices in deep-water breaking events; where the absence of turbulence-induced eddy viscosity results in the overprediction of the velocity and vorticity field in the breaking region.

Keywords:

non-hydrostatic wave model, breaking waves, wave-induced mean flow

\section{Introduction}

Surface wave breaking is a highly dissipative process, transferring energy and momentum into currents and turbulence, which enhances transport and mixing in the ocean surface layer and surf zone (Peregrine, 1983; Battjes, 1988; Banner \& Peregrine, 1993; Melville, 1996; Perlin et al., 2013). It is well-established that wave-breaking-induced turbulent coherent structures, such as obliquely descending eddies, play significant role in the transport of dispersed bubbles and sediment in individual breaking waves in a localized, short-term manner (Nadaoka et al., 1989; Watanabe et al., 2005; Ting \& Reimnitz, 2015). However, long-term transport of dispersed particles and passive tracers are mainly dependent on the structure of wavebreaking-driven mean circulation and their evolution. For example, large coherent vortices induced by individual whitecaps in deep and intermediate water (Rapp \& Melville, 1990; Pizzo \& Melville, 2013; Derakhti \& Kirby, 
2014a) as well as undertow, longshore and rip currents (Longuet-Higgins, 1970; Svendsen, 1984) and large-scale, vertical-core eddies (Peregrine, 1998; Clark et al., 2012) in the surf zone are fairly well-understood wave-breakinginduced organized motions. Such organized motions need to be reasonably resolved in any non-hydrostatic model to truly estimate long-term transport and mixing processes at field scales. The effect of Langmuir circulation cells should also be taken into account in deep-water mixing (McWilliams et al., 1997).

Because of available computational resources, the whole range of the relevant scales can not be resolved in a single high-resolution framework such as large-eddy simulations (LES) combined with the volume-of-fluid (VOF) method for free-surface tracking (Watanabe et al., 2005; Lakehal \& Liovic, 2011; Derakhti \& Kirby, 2014b, 2016; Zhou et al., 2014; Lubin \& Glockner, 2015). Recently, several three-dimensional (3-D) wave-resolving nonhydrostatic models based on Reynolds-averaged Navier-Stokes (RANS) equations using surface- and terrain-following grid have been developed for coastal applications (Lin \& Li, 2002; Zijlema et al., 2011; Bradford, 2011; Ma et al., 2012; Shirkavand \& Badiei, 2014). As opposed to the VOF/LES framework, the free surface is a single-valued function of horizontal location, and detailed near-surface processes during active breaking, such as turbulence generation and air entrainment, can not be resolved in this lower-resolution framework. Due to computational efficiency, however, this framework can be used to study long-term, $O$ (days), and large-scale, $O(100 \mathrm{~m} \approx 10 \mathrm{~km})$, 
wave-breaking-driven circulation as well as transport of dispersed particles and passive tracers.

In Part 1 (Derakhti et al., 2016b) of this paper, NHWAVE as described in Derakhti et al. (2016a), is shown to predict organized wave motions in terms of the evolution of the wave height and phase-averaged free surface elevations and organized wave velocity, spectral evolution as well as the secondand third-order wave statistics in depth-limited regular and irregular breaking waves as accurate as those predicted by typical VOF/RANS simulations, where vertical grid resolution in all simulated cases is at least an order of magnitude coarser than that of typical VOF-based simulations. It is also shown that the dispersive and nonlinear properties of different wave packet components before and after the break point, the overall wave height decay and spectral evolution in steepness-limited unsteady breaking waves are captured by the model.

Our goal here is to examine what level of detail of wave-breaking-induced turbulence and mean circulation can be reproduced by the non-hydrostatic RANS model NHWAVE as described by Derakhti et al. (2016a). We carefully examine NHWAVE predictions of wave-breaking-induced mean circulation in terms of wave setup and undertow current in depth-limited breaking waves, and in terms of low-frequency forced waves and wave-breaking-driven largescale vortex structures in steepness-limited unsteady breakers. Further, the spatio-temporal evolution of wave-breaking-induced turbulence statistics in terms of turbulent kinetic energy is compared with corresponding measure- 
ments and the results of the VOF/LES simulation of Derakhti (2016). In addition, the effect of modeled turbulent eddy viscosity, $\nu_{t}$, on the predicted time-averaged velocity distribution in the surf zone is discussed.

The paper is organized as follows. A brief summary of the model set-up and choice of experimental conditions is given in $\S 2$. Results for depth-limited and steepness-limited breaking waves are presented in $\S 3$ and $\S 4$ respectively. In $\S 5$, the relative importance of $\nu_{t}$ in the predicted undertow profiles are discussed. Conclusions are presented in $\S 6$.

\section{Model set-up and choice of experimental conditions}

To avoid unnecessary duplication, the reader is referred to Part 1 (Derakhti et al., 2016b) for a brief description of governing equations and dynamic surface boundary conditions. A detailed description of the governing equations and boundary conditions may be found in Derakhti et al. (2016a). Details of the numerical method may be found in Ma et al. (2012) and Derakhti et al. (2015). In this section, the choice of experimental conditions is briefly explained.

We consider model performance for the case of regular and irregular depth-limited wave breaking on planar and barred beaches using the data sets of Ting \& Kirby (1994) and Scott et al. (2004) as well as for the case of steepness-limited unsteady wave breaking using the data set of Rapp \& Melville (1990). Table 1 summarizes the input parameters for the simulated

cases. Details of numerical set-up and inflow boundary conditions for the 
depth-limited breaking cases and the steepness-limited breaking case considered here are presented in $\S 3.1$ and $\S 4.1$ of Part 1, respectively.

Both spilling breaking (hereafter referred as TK1) and plunging breaking (hereafter referred as TK2) cases of Ting \& Kirby (1994) are selected to examine the model prediction of the temporal evolution of the phase-averaged velocity and turbulent kinetic energy, $k$, in depth-limited breaking waves. This experiment has been widely used by other researchers to validate both non-hydrostatic models using surface- and terrain-following grid (Ma et al., 2014; Bradford, 2011, 2012; Smit et al., 2013; Shirkavand \& Badiei, 2014) and VOF-based numerical models (Ma et al., 2011; Lin \& Liu, 1998a,b; Bradford, 2000; Christensen, 2006; Lakehal \& Liovic, 2011). The predicted time-averaged velocity and $k$ fields for TK1, TK2 and the regular breaking case of Scott et al. (2004) (hereafter referred as S1) are compared with corresponding experiments. Besides the measurements of Ting \& Kirby (1994), the model results also compared with the results of the VOF/LES simulation of Derakhti (2016). The latter follows the experimental set-up of Ting \& Nelson (2011) which is similar to TK1, as summarized in Table 1. Further, the predicted time-averaged velocity and $k$ fields for the irregular depth-limited cases of Mase \& Kirby (1992) and Scott et al. (2004) are compared with the simulated regular cases.

The model results for one of the unsteady plunging breakers of Rapp \& Melville (1990) (hereafter referred as RM1) in an intermediate-depth regime with $k_{c} d \approx 1.9$ are presented, where $k_{c}$ is the wave number of the center fre- 
Table 1: Input parameters for the simulated surf zone regular breaking cases on a planar beach. Here, $h_{0}$ is the still water depth in the constant-depth region, $H$ and $T$ are the wave height and period of the cnoidal wave generated by the wavemaker, $(k H)_{0}$ is the corresponding deep-water wave steepness of the generated wave, $\xi_{0}=s / \sqrt{H_{0} / L_{0}}$ is the self similarity parameter, and $s$ is the plane slope. For $\mathrm{S} 1$ and $\mathrm{S} 2, s$ is the averaged slope before the bar, assumed as $s \sim 1 / 12$. For the irregular wave cases, $T=T_{p}$ and $k=k_{p}$, where $p$ refers to the peak frequency of the incident waves. For S2, $H=H_{s 0}$ is the deepwater characteristic wave height. For the rest of irregular wave cases, $H=H_{r m s}$ at the first wave gauge.

\begin{tabular}{rccccccc}
\hline Case no. & $\begin{array}{c}H \\
(\mathrm{~m})\end{array}$ & $\begin{array}{c}T \\
(\mathrm{~s})\end{array}$ & $(k H)_{0}$ & $k_{0} h_{0}$ & $\xi_{0}$ & $\begin{array}{c}\text { breaking } \\
\text { type }\end{array}$ & Exp. \\
\hline$T K 1$ & 0.125 & 2.0 & 0.126 & 0.6 & 0.20 & regular, spilling & Ting \& Kirby (1994) \\
$\sim T K 1$ & 0.122 & 2.0 & 0.119 & 0.5 & 0.22 & regular, spilling/weakly plunging & Ting \& Nelson (2011) \\
$T K 2$ & 0.128 & 5.0 & 0.015 & 0.2 & 0.59 & regular, plunging & Ting \& Kirby (1994) \\
$S 1$ & 0.64 & 4.0 & 0.148 & 1.2 & 0.52 & regular, plunging & Scott et al. $(2004)$ \\
$M K 1$ & 0.04 & 1.7 & 0.058 & 0.9 & 0.52 & irregular, plunging & Mase \& Kirby $(1992)$ \\
$M K 2$ & 0.04 & 1.0 & 0.161 & 2.0 & 0.31 & irregular, spilling & Mase \& Kirby $(1992)$ \\
$S 2$ & 0.59 & 4.0 & 0.136 & 1.2 & 0.54 & irregular, plunging & Scott et al. $(2004)$ \\
\hline
\end{tabular}

quency wave of the input packet as defined in $\S 4.1$ of Part 1 . The evolution of the mean velocity field and large mean vortex in case RM1 are compared with the corresponding measurements and the results of the VOF/LES simulation of Derakhti \& Kirby (2014a). In this experiment, breaking waves were generated using the dispersive focusing technique, in which an input packet propagates over an constant depth and breaks at a predefined time, $t_{b}$, and location, $x_{b}$. The input parameters for the simulated case are summarized in Table 2 .

\section{Depth-limited breaking waves}

For regular wave cases, \langle\rangle and $\overline{(~)}$ refer to phase and time averaging over five subsequent waves after the results reach quasi-steady state, respectively. We found that averaging over more than five waves results in approximately 
Table 2: Input parameters for the simulated focused wave packets. $h$ is the still water depth, $S=\sum_{i=1}^{N} a_{i} k_{i}$ is the global steepness, $N$ is the number of components in the packet, $a_{i} k_{i}$ is the component steepness which is the same for the all components, and the discrete frequencies $f_{i}$ were uniformly spaced over the band $\Delta f=f_{N}-f_{1}$ with a central frequency defined by $f_{c}=\frac{1}{2}\left(f_{N}+f_{1}\right)$.

\begin{tabular}{ccccccc}
\hline Case no. & $\begin{array}{c}h \\
(\mathrm{~m})\end{array}$ & $S$ & $\begin{array}{c}f_{c} \\
(1 / \mathrm{s})\end{array}$ & $\Delta f / f_{c}$ & $\mathrm{~N}$ & $\begin{array}{c}\text { breaking } \\
\text { type }\end{array}$ \\
\hline$R M 1$ & 0.60 & 0.352 & 0.88 & 0.73 & 32 & plunging \\
\hline
\end{tabular}

similar statistics in the shoaling and surf zone regions. The corresponding measured averaged variables were calculated by averaging over 102 successive waves starting at a minimum of 20 minutes after the initial wavemaker movement for TK1 and TK2, and by phase averaging over 150 successive waves and ensemble averaging over at least 8 realizations for S1. For irregular wave cases, $\overline{()}$ refers to long-time averaging over several minutes, or more than 250 waves. The mean water level is defined as $\bar{D}=h+\bar{\eta}$, where $h$ is the still water depth and $\bar{\eta}$ is the wave-induced set-down/set-up.

\subsection{Time-averaged water surface level and velocity field}

Comparing with observations, Figure 1 shows that the model accurately predicts the evolution of the time-averaged (mean) water surface level $\bar{\eta}$ from outside of the surf zone up to the swash zone. In particular, the maximum wave set-up is accurately predicted in all cases except in S2, indicating that NHWAVE accurately predicts the gradient of the wave radiation stress in both spilling and plunging depth-limited breaking waves. As shown in panel (a), the jump in the observed set-up in the spilling case TK1 at $x \approx 5 \mathrm{~m}$ does 
not exist in the predicted results; the same discrepancy was also reported in other studies such as Smit et al. (2013, Figure 7) and Tonelli \& Petti (2010, Figure 9). In S1, the mean water level is accurately resolved from deep water up to the swash zone, as apposed to the VOF/RANS simulation of Jacobsen et al. (2014, Figure 4B) which overpredicts wave-induced set-up after the bar.

Figure 2 shows the NHWAVE prediction of the spatial distribution of the time-averaged velocity field using 4,8 and 16 vertical $\sigma$-levels for the spilling case TK1 and that by the 3-D VOF/LES simulation of Derakhti (2016). To obtain the Eulerian mean velocities, the model results in the $\sigma$ coordinate system first were interpolated onto a fixed vertical mesh at each cross-shore location using linear interpolation, and then time averaging was performed. The results of the simulations with various vertical resolutions have approximately the same structure in the surf zone. The predicted return current using $4 \sigma$-levels shown in panel $(a)$ has not detached from the bed at $X \sim 0$ in contrast to the simulations with 8 and $16 \sigma$-levels and the 3-D VOF/LES simulation shown in panel $(d)$. Regardless of the various vertical resolutions, the predicted time-averaged velocity in the transition region has a relatively stronger vertical variation compared with the 3-D VOF/LES simulation. A similar pattern of results was found for the plunging case TK2 and is not shown. Figures 3 and 4 show that the slope in the predicted undertow profiles in the transition region is larger than in the measured undertow profiles for both cases. This difference is more noticeable in the 
plunging case TK2, in which the measured profiles are approximately uniform with depth. The normalized undertow current for the irregular wave cases MK1 and MK2 (not shown) have smaller magnitude than that for regular wave cases TK1 and TK2 with the same vertical structures within the surf zone. This is consistent with the measurements of Ting (2001) which has the similar incident wave conditions and experimental set-up compared with the simulated irregular breaking waves on a planner beach in the present study. Figure 5 shows the spatial distribution of the time-averaged velocity field using $8 \sigma$-levels for the depth-limited regular (S1) and irregular (S2) breaking waves on a barred beach. As in TK1, the predicted return current using 4 $\sigma$-levels (not shown) has not detached from the bed shoreward of the breaking point, as opposed to the simulation with $8 \sigma$-levels shown in Figure 5(a). In $\mathrm{S} 1$, the results of the simulations with 4 (not shown) and $8 \sigma$-levels have approximately the same structure after the breaking point, where the predicted undertow current using $8 \sigma$-levels has larger magnitude in the entire surf zone. In S2, the predicted undertow current using 4 (not shown) and 8 $\sigma$-levels (Figure 5b) have approximately the same structure and magnitude in the entire surf zone. The magnitude of undertow in the irregular case S2 is smaller than that in the regular case S1; a similar trend was observed in the simulated cases on a planar beach. The slope of the undertow profile in S1 has a strong spatial variation near the break point as shown in Figure 6(c), where the slope of the undertow profile at $x=48.0 \mathrm{~m}$ (red lines) considerably decreases compared with that at $x=51.0 \mathrm{~m}$ (black lines). This is due to the 
detachment of the undertow current from the bed, forming negative slopes at seaward of the break point. Figure 6(c) also shows that the model predicts breaking seaward of the measured break point as in TK1 and TK2. The velocity measurements were carried out at seven cross-shore locations, shown in Figure 5(a), using Acoustic Doppler Velocimeters (ADVs) sampling at $50 \mathrm{~Hz}$. The measured undertow profiles at two different longshore locations (shown by open and solid circles) reveal that the time-averaged velocity field varies in the spanwise direction close to the break point; the 3-D effects are absent in our two-dimensional simulation.

Comparing the results with the measured undertow profiles, it is seen that the undertow current on top of and after the bar as well as in the inner surf zone are fairly reasonably captured using as few as $4 \sigma$-levels. Hereafter, we use the statistical method developed by Willmott (1981) to asses the model predictive skill for various breaking-related properties. The model predictive skill is given by

$$
\text { Skill }=1-\frac{\sum_{i=1}^{N}\left|\psi_{i, \mathrm{sim}}-\psi_{i, \mathrm{obs}}\right|^{2}}{\sum_{i=1}^{N}\left[\left|\psi_{i, \mathrm{sim}}-\bar{\psi}_{\mathrm{obs}}\right|+\left|\psi_{i, \mathrm{obs}}-\bar{\psi}_{\mathrm{obs}}\right|\right]^{2}},
$$

where $\psi$ is the variable being compared, $\bar{\psi}$ is its sample mean value, and subscripts ()$_{\text {sim }}$ and ()obs stand for the simulated results and observations, respectively. This skill parameter measures the degree to which the observed deviations about the observed mean correspond with the predicted deviations about the observed mean. Perfect agreement between the simulated results 
and observations yields a skill of 1.0 whereas complete disagreement yields a skill of 0 (Willmott, 1981). In the spilling case TK1, the model predictive skills using $8 \sigma$-levels for the undertow profiles varies from 0.7 near the initial break point up to 0.95 in the inner surf zone. In the plunging case TK2, the predictive skills are between 0.8 and 0.9 . As apposed to TK1, the model skill for $\bar{u}$ decreases at shallower depths. In S1, the model shows predictive skills higher than 0.8 on the top of and shoreward face of the bar (Figure $6 d, e, f$ ), while in other regions the predicted undertow profiles have an opposite slope compared with observations resulting in the predictive skills lower than 0.2. In all cases, the increase of vertical resolution from 4 to $8 \sigma$-levels improves the corresponding model skill especially in the outer surf zone, while the change in the predictive skills due to further increase of vertical resolution from 8 to $16 \sigma$-levels is negligibly small. A relatively larger slope in the predicted undertow profiles compared with observations is discussed in $\S 5$.

\subsection{Turbulence Statistics}

Figure 7 shows snapshots of the predicted instantaneous turbulent kinetic energy $k$ distribution using 4, 8 and $16 \sigma$-levels for TK1 and that by the 3-D VOF/LES simulation of Derakhti (2016). In general, the overall distribution of $k$ using various vertical resolutions is the same. However, increasing the vertical resolution decreases the predicted $k$ levels near the wave front region. The same trend is also observed for TK2 (not shown). Further, the predicted $k$ levels above the trough levels are smaller than the 3-D VOF/LES simula- 
tion, mainly due to the absence of near-surface turbulence generation in the present study.

Figure 8 shows a comparison of measured and simulated phase-averaged turbulent kinetic energy $\langle k\rangle$ time series close to the bed and trough level at different cross-shore locations using 4,8 and $16 \sigma$-levels for the spilling case TK1. $\langle k\rangle$ is overestimated higher in the water column during the entire wave period especially close to the break point. This overestimation has been also reported in previous VOF-based $k-\epsilon$ studies (Lin \& Liu, 1998a; Ma et al., 2011). Lin \& Liu (1998a) argued that this is because the RANS simulation can not accurately predict the initiation of turbulence in a rapidly distorted shear flow such as breaking waves. Alternately, Ma et al. (2011) incorporated bubble effects into the conventional single phase $k-\epsilon$ model, and concluded that the exclusion of bubble-induced turbulence suppression is the main reason for the overestimation of turbulence intensity by single phase $k-\epsilon$. Comparing Figure 8 with the corresponding results from the VOF-based model Ma et al. (2011, Figure 7), we conclude that predicted $\langle k\rangle$ values below trough levels in spilling breaking waves by NHWAVE using as few as $4 \sigma$-levels are at least as accurate as the VOF-based simulation without bubbles.

In the plunging case TK2, a different behavior is observed in the predicted $\langle k\rangle$ values shown in Figure 9 compared with the corresponding results for the spilling case TK1, regardless of the various vertical resolutions. After the initial break point, $\langle k\rangle$ is underpredicted especially for lower elevations. 
The model could not resolve the sudden injection of $k$ into the deeper depths at the initial stage of active breaking and thus, there is a considerable underprediction of $\langle k\rangle$ at the beginning of active breaking below trough level.

The NHWAVE predictions of near-bed and above-trough time-averaged turbulent kinetic energy $\bar{k}$ are smaller than the corresponding $\bar{k}$ predictions by the 3-D VOF/LES simulation (not shown). The increase of the vertical resolution leads to a more concentrated patch of $\bar{k}$ in TK1; a similar trend is also observed for TK2 (not shown). Figures 10, 11 and 12 show the comparison of modeled and measured $\bar{k}$ profiles at different cross-shore locations before and after the initial break point for TK1, TK2 and S1 respectively. In the plunging case TK2, the noticeable underprediction of $\langle k\rangle$ at the initial stage of active breaking shown in Figure 9 compensates relatively smaller overprediction of $\langle k\rangle$ at the other phases, resulting to apparent smaller $\bar{k}$ values than those in the measurement in the shoreward end of the transition region and inner surf zone, as shown in Figure 11(B-D). In $\mathrm{S} 1$, the model accurately predicts the cross-shore variation of $\bar{k}$, with the large $\bar{k}$ levels across the breaker bar, where the waves are breaking, and the subsequent decay of $\bar{k}$ level on the seaward face as well as after the bar. In the spilling case TK1, the model predictive skills for $\bar{k}$ varies from 0.2 close to the initial break point up to 0.9 in the inner surf zone using as few as $4 \sigma$-levels, where the skill values continuously increase across the transition region and inner surf zone. In the plunging case TK2, however, the corresponding model skills are lower than 0.6 in the surf zone regardless of various vertical resolutions; 
with the minimum value of 0.2 in the inner surf zone. In S1, the model skills are between 0.7 and 0.9 across and shoreward of the breaker bar.

Ting (2001) observed that the mean of the highest one-third wave-averaged $k$ values in his irregular waves in the middle surf zone was about the same as $\bar{k}$ in a regular wave case TK1, where deep-water wave height to wavelength ratio of those two cases was on the same order. Here, results show that the values of $\sqrt{\bar{k} / g \bar{D}}$ for the irregular wave case MK2 (not shown) are at the same order or even larger than those in regular breaking cases in the middle and inner surf zone. In the outer surf zone, however, the values of $\sqrt{\bar{k} / g \bar{D}}$ are smaller than those in regular breaking cases. Although the $\bar{k}$ values decrease near the bottom in the outer surf zone similar to regular breaking cases, they have small vertical and cross-shore variations in the inner surf zone. Further, the predicted $\bar{k}$ using 4 and $8 \sigma$-levels have approximately the same structure and magnitude in the entire surf zone in all cases.

\section{Steepness-limited unsteady breaking waves}

In this section, the normalized time and locations are defined as

$$
x^{*}=\frac{x-x_{o b}}{L_{c}}, \quad z^{*}=\frac{z}{L_{c}}, \quad t^{*}=\frac{t-t_{o b}}{T_{c}},
$$

where $T_{c}$ and $L_{c}$ are the period and wavelength of the center frequency wave of the input packet, respectively. Here, $t_{o b}$ and $x_{o b}$ are the time and location at which the forward jet hits the free surface, obtained from the corresponding 
VOF simulations.

\subsection{Wave-breaking-induced mean circulation}

Comprehensive experimental work by Rapp \& Melville (1990) and Drazen

\& Melville (2009) has revealed the main characteristics of the ensembleaveraged flow field in unsteady breaking waves, especially after active breaking. The ensemble-averaged velocity field may be decomposed into

$$
\langle\mathbf{u}\rangle=\mathbf{u}_{w}+\mathbf{u}_{f w}+\mathbf{u}_{c},
$$

where $\mathbf{u}_{w}$ is the orbital velocity of the surface waves, $\mathbf{u}_{f w}$ is the velocity of the forced long-waves induced by breaking, and $\mathbf{u}_{c}$ is the Eulerian-mean current stemming from the momentum loss during the breaking and/or Stokes drift. Rapp \& Melville (1990) measured the velocity field using LDV at seven elevations and seven $x$ locations in the breaking region. The measured velocity signals are low-pass filtered using the threshold frequency of $0.3 \mathrm{~Hz}$, to remove the surface waves as in Rapp \& Melville (1990), where the frequency range of the input surface waves is $0.56<f_{i}<1.20 \mathrm{~Hz}$. Figure 13 shows the low-pass filtered results and the corresponding measurements for RM1 at $x^{*}=0.15$ and $x^{*}=0.60$, from very close to the free surface to $z^{*}=-0.15$ $(\approx z=-d / 2)$. The smaller low-passed filtered velocity field is due to the smaller wave dissipation and smaller wave forcing, predicted by the model. The Eulerian-mean current can be calculated by time averaging of the 
ensemble-averaged velocity signal,

$$
\mathbf{u}_{c}=\overline{\mathbf{u}}=\frac{1}{t_{2}^{*}-t_{1}^{*}} \int_{t_{1}^{*}}^{t_{2}^{*}}\langle\mathbf{u}\rangle d t^{*}
$$

where $t_{1}^{*}$ and $t_{2}^{*}$ cover the entire wave packet. During time integration for each grid point, when the point is above the free surface the velocity signal is zero. Figure 14 shows the spatial distribution of the normalized mean current and its horizontal-averaged between $x^{*}=0$ and 1.5, as well as the normalized horizontal-averaged mass flux below the depth $z^{*}, \widehat{M^{*}}\left(z^{*}\right)=\int_{z_{1}^{*}}^{z^{*}} \widehat{u_{c}^{*}} d z^{*}$ where $z_{1}^{*}=-0.31$ is the bottom elevation, for RM1 using $8 \sigma$-levels (top panels) together with the VOF/LES results by Derakhti \& Kirby (2014a) (bottom panels). The positive current near the surface, the return negative current at lower depths and the two distinct circulation cells are captured by the model as in the VOF/LES results. Comparing with the measurements of (Rapp \& Melville, 1990, Figure 43) and the VOF/LES simulation, we can see that the model generated a large mean vortex with relatively stronger velocity field. We believe this is due to the absence of an enhanced eddy viscosity that would be present as a result of the turbulence, which was not captured by NHWAVE in unsteady breaking cases. In addition, the model predicts relatively larger cells than those predicted by the VOF/LES simulation, especially in the $x$ direction. The predicted patch of persistent vorticity (not shown) is consistent with Drazen \& Melville (2009, Figure 4) and the VOF/LES simulation of Derakhti \& Kirby (2014a, Figure 4.16), 
having larger vorticity values due to underestimation of effective viscosity in the absence of turbulence.

\section{Discussion}

\subsection{Effects of eddy viscosity on undertow profiles}

In this section, we examine why the model predictions of undertow profiles, shown in $\S 2$, had a relatively larger slope compared with observations in the transition region of the depth-limited breaking waves, where the discrepancy was more pronounced in the plunging breakers.

Considering available undertow models using an eddy viscosity closure scheme (see Garcez Faria et al., 2000, among others), it is known that the three factors determine the vertical profile of undertow currents; including (i) bottom boundary layer (BBL) processes, leading to a landward streaming velocity (Longuet-Higgins, 1953; Phillips, 1977) or a seaward streaming velocity due to a time-varying eddy viscosity within the wave turbulent BBL (Trowbridge \& Madsen, 1984), close to the bed; (ii) vertical variations of the eddy viscosity $\nu_{t}$, affected mainly by breaking-generated turbulence; and (iii) wave forcing due to the cross-shore gradients of radiation stress, setup, and convective acceleration of the depth-averaged flow. As explained by Garcez Faria et al. (2000), the slope of the undertow profile is a function of both wave forcing and $\nu_{t}$. Large values of wave forcing generates more vertical shear, resulting in a parabolic profile, whereas large values of $\nu_{t}$ reduce vertical shear, leading to a more uniform velocity profile with depth. 
Figure 15 shows the predicted undertow profiles using $8 \sigma$-levels for the regular depth-limited spilling breaking TK1 and plunging breaking TK2 cases together with the corresponding measurements, where the different model predictions are obtained by using constant $(0,100 \nu, 500 \nu$, and $1000 \nu, \nu=$ $10^{-6} \mathrm{~m}^{2} / \mathrm{s}$ ) and spatio-temporal varying $\nu_{t}$ calculated based on the $k-\epsilon$ turbulence model. As the magnitude of $\nu_{t}$ increases the slope of the undertow profiles significantly decreases in both cases. Figure 16 shows the snapshots of the distribution of modeled $\nu_{t}$ for TK1 using 4,8 and $16 \sigma$-levels. Far behind each bore-front region, for example $1<X<3$, the spatial structure of the predicted $\nu_{t}$ is similar to that reported in the VOF/RANS simulation in Lin \& Liu (1998a, Figures 12c and 13c ), where the magnitude of the former is up to $30 \%$ smaller than the latter. Close to the bore-front regions, the NHWAVE predicted values of $\nu_{t}$ are significantly smaller than those reported in Lin \& Liu (1998a, Figures 12c and 13c), where increasing vertical resolution results in a greater deviation compared with the VOF/RANS simulation.

Compared with observations, we conclude that the underprediction of modeled $\nu_{t}$ results in greater vertical shear in the predicted undertow profiles, where the larger discrepancy in TK2 is due to a more noticeable underprediction of $\nu_{t}$ in TK2 compared with that in TK1. In addition, the lack of second-order BBL effects, and thus the absence of the associated streaming velocity in the present simulations may explain some part of the difference between the predicted and measured return current close to the bed. 


\subsection{Computational efficiency and CPU timing}

In this section, we compare the computational cost of the non-hydrostitic $\sigma$-coordinate RANS model NHWAVE with our high-resolution VOF/LES model Derakhti \& Kirby (2014b) for the regular depth-limited spilling breaking waves case TK1. The same horizontal grid resolution and Courant number are used in all simulations. Various vertical resolutions of 4,8 and 16 $\sigma$-layers are considered for the NHWAVE simulations. Both NHWAVE and VOF/LES model use the HYPRE parallel solver and preconditioner library to solve the linear systems. All simulations are run for a 100s simulation time on the same HPC cluster by using 20 processors.

As summarized in Table 3, the NHWAVE simulation with $4 \sigma$-layers are about two order of magnitude faster than the corresponding single-phase VOF/LES simulation. If we assume that the required horizontal resolution for an NHWAVE simulation can be coarser than that for a VOF/LES simulation by a factor of $r$, the speed ratio of the required CPU-time between two simulations increases by an additional factor of $r^{3}$. Such a considerable decrease in the required computational resources for a typical NHWAVE simulation compared with its comparable VOF/LES simulation makes the NHWAVE model suitable for field-modeling of wave-breaking-induced turbulence and mean circulation, providing vertical structure of 3-D velocity field and turbulence statistics over the inner shelf and nearshore regions. 
Table 3: Normalized CPU-time of the simulations by the $\sigma$-coordinate RANS model NHWAVE using various vertical resolutions and the VOF/LES model Derakhti \& Kirby (2014b) for the regular depth-limited spilling breaking waves case TK1. The reference value is the CPU time of the NHWAVE simulation with $4 \sigma$-layers for a 100s simulation time, which is equal to 13 procs.hour.

\begin{tabular}{lc}
\hline Cases & $R_{C P U}$ \\
\hline NHWAVE, $4 \sigma$-layers & 1 \\
NHWAVE, $8 \sigma$-layers & 1.7 \\
NHWAVE, 16 $\sigma$-layers & 3 \\
VOF/LES, multi-layer & 91 \\
\hline
\end{tabular}

\section{Conclusions}

In this paper, we examined wave-breaking predictions ranging from shallowto deep-water conditions using a 3-D non-hydrostatic $\sigma$-coordinate RANS model, NHWAVE (Ma et al., 2012), comparing results both with corresponding experiments and with the results of a VOF/Navier-Stokes solver (Ma et al., 2011; Derakhti \& Kirby, 2014a; Derakhti, 2016). The new version of NHWAVE has been described in Derakhti et al. (2016a), including the new governing equations and consistent surface and bottom boundary conditions. We considered regular and irregular depth-limited breaking waves on planar and barred beaches as well as steepness-limited unsteady breaking focused wave packets in intermediate and deep depths. The same equations and numerical methods were used for the various depth regimes and involved no ad-hoc treatment, such as imposing hydrostatic conditions, in triggering breaking. In Part 1 (Derakhti et al., 2016b) of this paper, we showed that NHWAVE, as described in Derakhti et al. (2016a), accurately captured or- 
ganized wave motions in terms of free-surface evolution, spectral evolution and wave statistics as accurate as those predicted by typical VOF/RANS simulations, where vertical grid resolution in all simulated cases was at least an order of magnitude coarser than that of typical VOF-based simulations. In Part 2, we examined the model reproduction of wave-breaking-induced turbulence and mean circulation. The main conclusions of the present study can be categorized as follows:

(a) Depth-limited breaking waves: using as few as $4 \sigma$-levels, the model was shown to fairly reasonably predict the evolution of the wave-induced set-up as well as the evolution of the ensemble-averaged turbulent kinetic energy, $k$, distribution under trough levels for depth-limited breaking waves both on a planar and barred beaches as accurate as those predicted by typical VOF/RANS simulations without bubble effects. Under strong plunging breakers, the rapid advection of high $k$ to lower depths can not captured by the model due to the unresolved jet impact and subsequent splash processes. Further, the turbulent eddy viscosity $\nu_{t}$ was underestimated in both spilling and plunging breakers, especially in the breaking crest regions. The underestimation of $\nu_{t}$ was shown to result in the overprediction of the slope of undertow profiles, especially in the transition region. We believe that both the underprediction of $k$ in plunging breakers and the underprediction of $\nu_{t}$ are mainly due to the ignorance of turbulent stress at the free surface in borefront regions, which the model assumes to be smooth, rather than a selected number of vertical $\sigma$-levels. The $k$ prediction above trough levels and the 
$\nu_{t}$ estimation near crest regions may be further improved by replacing the zero-tangential-stress boundary condition or the zero-gradient boundary condition for $k$ with physics-based models such as those proposed by Brocchini \& Peregrine (2001) and Brocchini (2002).

(b) Steepness-limited breaking waves: Although the absence of turbulenceinduced eddy viscosity in unsteady breaking waves predictions led to the overprediction of the velocity and vorticity field in the breaking region, the model was shown to predict the structures of the mean velocity field including large breaking-induced coherent vortices. The near-surface turbulence model for whitecap events, e.g., the model proposed by Brocchini (2002) to set a boundary condition for $k$, is needed to provide sufficient $k$ levels during active breaking, with which the model will produce the turbulence field, leading to an enhance eddy viscosity and an appropriate amount of breaking-induced dissipation in the breaking region.

\section{Acknowledgments}

The authors gratefully thank Zhigang Tian, Marc Perlin, and Wooyoung Choi for providing the deep water laboratory data. This work was supported by ONR, Littoral Geosciences and Optics Program (grant N00014-131-0124); NSF, Physical Oceanography Program (grant OCE-1435147); and through the use of computational resources provided by Information Technologies at the University of Delaware. 
Banner, M. L. \& Peregrine, D. H. 1993 Wave breaking in deep water. Ann. Rev. Fluid Mech. 25, 373-397.

Battues, J. A. 1988 Surf-zone dynamics. Ann. Rev. Fluid Mech. 20, 257291.

Bradford, S. F. 2000 Numerical simulation of surf zone dynamics. $J$. Waterway, Port, Coastal, Ocean Eng. 126, 1-13.

Bradford, S. F. 2011 Nonhydrostatic model for surf zone simulation. $J$. Waterway, Port, Coastal, Ocean Eng. 137, 163-174.

Bradford, S. F. 2012 Improving the efficiency and accuracy of a nonhydrostatic surf zone model. Coastal Eng. 65, 1-10.

Brocchini, M. 2002 Free surface boundary conditions at a bubbly/weakly splashing air-water interface. Phys. Fluids 14, 1834-1840.

Brocchini, M. \& Peregrine, D. H. 2001 The dynamics of strong turbulence at free surfaces. part 2. free-surface boundary conditions. J. Fluid Mech. 449, 255-290.

Christensen, E. D. 2006 Large eddy simulation of spilling and plunging breakers. Coastal Eng. 53, 463-485.

Clark, D. B, Elgar, S. \& Raubenheimer, B. 2012 Vorticity generation by short-crested wave breaking. Geophys. Res. Lett. 39 . 
DERAKHTI, M. 2016 LES and $\sigma$-coordinate RANS simulations of laboratory surface wave breaking. PhD thesis, University of Delaware.

Derakhti, M. \& Kirby, J. T. 2014a Bubble entrainment and liquidbubble interaction under unsteady breaking waves. Tech. Rep.. CACR-1406, Center for Applied Coastal Research, Dept. of Civil \& Environmental Engineering, University of Delaware.

Derakhti, M. \& Kirby, J. T. $2014 b$ Bubble entrainment and liquidbubble interaction under unsteady breaking waves. J. Fluid Mech. 761, $464-506$.

Derakhti, M. \& Kirby, J. T. 2016 Breaking-onset, energy and momentum flux in focused wave packets. J. Fluid Mech. 790, 553-581.

Derakhti, M., Kirby, J. T., Shi, F. \& MA, G. 2015 NHWAVE: Model revisions and tests of wave breaking in shallow and deep water. Tech. Rep.. CACR-14-18, Center for Applied Coastal Research, Dept. of Civil \& Environmental Engineering, University of Delaware.

Derakhti, M., Kirby, J. T., Shi, F. \& MA, G. 2016a NHWAVE: Consistent boundary conditions and turbulence modeling. Ocean Mod., under review .

Derakhti, M., Kirby, J. T., Shi, F. \& MA, G. $2016 b$ Wave breaking from surf zone to deep water in a non-hydrostatic RANS model. Part 1: Organized wave motions. Ocean Mod. under review . 
Drazen, D. A. \& Melville, W. K. 2009 Turbulence and mixing in unsteady breaking surface waves. J. Fluid Mech. 628, 85.

Garcez Faria, A. F., Thornton, E. B., Lippmann, T. C. \& StanTON, T. P. 2000 Undertow over a barred beach. J. Geophys. Res. 105, 16999-17010.

Jacobsen, N. G, Fredsoe, J. \& Jensen, J. H 2014 Formation and development of a breaker bar under regular waves. part 1: Model description and hydrodynamics. Coastal Eng. 88, 182-193.

Lakehal, D. \& Liovic, P. 2011 Turbulence structure and interaction with steep breaking waves. J. Fluid Mech. 674, 522-577.

Lin, P. \& Li, C. W. 2002 A $\sigma$-coordinate three-dimensional numerical model for surface wave propagation. Int. J. Num. Methods Fluids 38, 10451068.

Lin, P. \& LiU, P.L.-F 1998a A numerical study of breaking waves in the surf zone. J. Fluid Mech. 359, 239-264.

Lin, P. \& LiU, P.L.-F $1998 b$ Turbulence transport, vorticity dynamics, and solute mixing under plunging breaking waves in surf zone. J. Geophys. Res 103, 667-694.

Longuet-Higgins, M. S. 1953 Mass transport in water waves. Phil. Trans. Roy. Soc. London, A 245, 535-581. 
Longuet-Higgins, M. S. 1970 Longshore currents generated by obliquely incident sea waves: 1. J. Geophys. Res. 75, 6778-6789.

Lubin, P. \& Glockner, S. 2015 Numerical simulations of threedimensional plunging breaking waves: generation and evolution of aerated vortex filaments. J. Fluid Mech. 767, 364-393.

MA, G., Chou, Y. \& Shi, F. 2014 A wave-resolving model for nearshore suspended sediment transport. Ocean Mod. 77, 33-49.

MA, G., Shi, F. \& Kirby, J. T. 2011 A polydisperse two-fluid model for surf zone bubble simulation. J. Geophys. Res.: Oceans 116, C05010, doi:10.1029/2010JC006667.

MA, G., ShI, F. \& Kirby, J. T. 2012 Shock-capturing non-hydrostatic model for fully dispersive surface wave processes. Ocean Mod. 43, 22-35.

Mase, H. \& Kirby, J. T. 1992 Hybrid frequency-domain KdV equation for random wave transformation. In Proc. 23d Int. Conf. Coastal Eng., pp. 474-487. Venice.

McWilliams, J. C., Sullivan, P. P. \& Moeng, C.-H. 1997 Langmuir turbulence in the ocean. J. Fluid Mech. 334, 1-30.

Melville, W. K. 1996 The role of surface-wave breaking in air-sea interaction. Ann. Rev. Fluid Mech. 28, 279-321. 
Nadaoka, K., Hino, M. \& Koyano, Y. 1989 Structure of the turbulent flow field under breaking waves in the surf zone. J. Fluid Mech 204, 359387.

Peregrine, D. H. 1983 Breaking waves on beaches. Ann. Rev. Fluid Mech. 15, 149-178.

Peregrine, D. H. 1998 Surf zone currents. Theor. comput. fluid dyn. 10, 295-309.

Perlin, M., Choi, W. \& Tian, Z. 2013 Breaking waves in deep and intermediate waters. Ann. Rev. Fluid Mech. 45, 115-145.

Phillips, O. M. 1977 The dynamics of the upper ocean. Cambridge University Press, London.

Pizzo, N. E. \& Melville, W. K. 2013 Vortex generation by deep-water breaking waves. J. Fluid Mech. 734, 198-218.

Rapp, R. J. \& Melville, W. K. 1990 Laboratory measurements of deepwater breaking waves. Phil. Trans. Roy. Soc. A, 331, 735-800.

Scott, C. P, Cox, D. T, Shin, S. \& Clayton, N. 2004 Estimates of surf zone turbulence in a large-scale laboratory flume. In Proc. 29th Int. Conf. Coastal Eng., Portugal, pp. 379-391. 
Shi, F., Kirby, J. T., Harris, J. C, Geiman, J. D \& Grilli, S. T 2012 A high-order adaptive time-stepping TVD solver for Boussinesq modeling of breaking waves and coastal inundation. Ocean Modelling 43, 36-51.

Shirkavand, A. \& Badiei, P. 2014 The application of a Godunov-type shock capturing scheme for the simulation of waves from deep water up to the swash zone. Coastal Eng. 94, 1-9.

Smit, P., Zijlema, M. \& Stelling, G. 2013 Depth-induced wave breaking in a non-hydrostatic, near-shore wave model. Coastal Eng. 76, 1-16.

Svendsen, I. A. 1984 Mass flux and undertow in a surf zone. Coastal Eng. $8,347-365$.

TinG, F. C. K. 2001 Laboratory study of wave and turbulence velocities in a broad-banded irregular wave surf zone. Coastal Eng. 43, 183-208.

Ting, F. C. K. \& KirBy, J. T. 1994 Observation of undertow and turbulence in a laboratory surf zone. Coastal Eng. 24, 51-80.

Ting, F. C. K. \& Nelson, J. R. 2011 Laboratory measurements of large-scale near-bed turbulent flow structures under spilling regular waves. Coastal Eng. 58, 151-172.

Ting, F. C. K. \& Reimnitz, J. 2015 Volumetric velocity measurements of turbulent coherent structures induced by plunging regular waves. Coastal Eng. 104, 93-112. 
${ }_{567}$ Tonelli, M. \& Petti, M. 2010 Finite volume scheme for the solution of 2d 568 extended boussinesq equations in the surf zone. Ocean Eng. 37, 567-582.

${ }_{569}$ Trowbridge, J. \& Madsen, O. S. 1984 Turbulent wave boundary layers: 570 2. second-order theory and mass transport. J. Geophys. Res. 89, 79995718007.

572 Watanabe, Y., Saeki, H. \& Hosking, R. J. 2005 Three-dimensional 573 vortex structures under breaking waves. J. Fluid Mech. 545, 291-328.

574 Willmott, C. J. 1981 On the validation of models. Phys. Geography 2, $575 \quad 184-194$.

576

577

578

579

580

581

Zhou, Z., Sangermano, J., Hsu, T. J. \& Ting, F. C. K. 2014 A numerical investigation of wave-breaking-induced turbulent coherent structure under a solitary wave. J. Geophys. Res.:Oceans 119, 6952-6973.

Zijlema, M., Stelling, G. \& Smit, P. 2011 SWASH: An operational public domain code for simulating wave fields and rapidly varied flows in coastal waters. Coastal Eng. 58, 992-1012. 

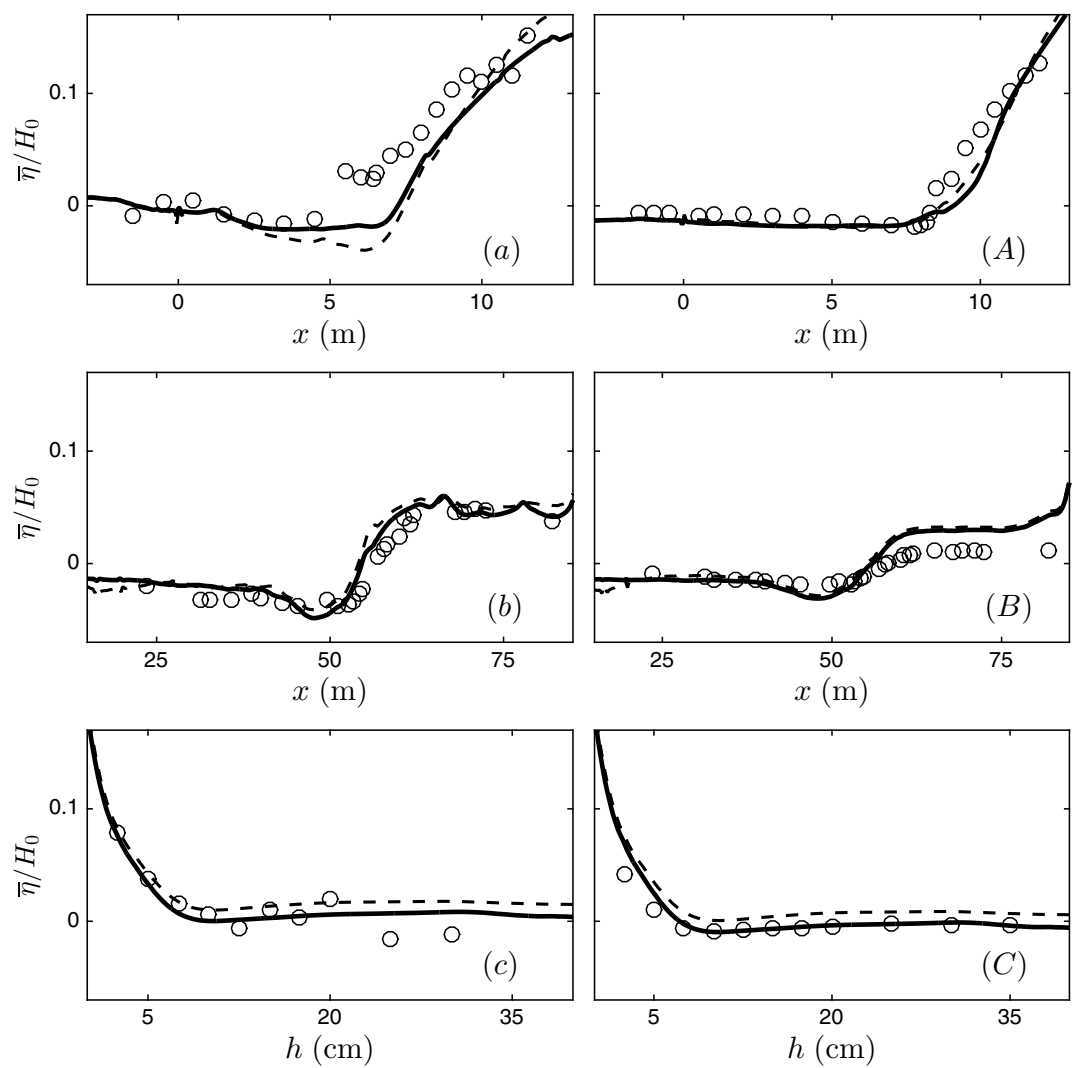

Figure 1: Cross-shore variation of wave-induced set-down/set-up for the regular depthlimited breaking cases $(a)$ TK1, $(A)$ TK2, and (b) S1; and irregular depth-limited breaking cases $(B) \mathrm{S} 2,(c) \mathrm{MK} 1$, and $(C)$ MK2. Comparison between NHWAVE results with $4 \sigma$ levels (dashed lines) and $8 \sigma$-levels (solid lines), and the corresponding measurements (circle markers). Regular waves in TK1, TK2 and S1 were observed to break at $x=6.4 \mathrm{~m}$, $7.8 \mathrm{~m}$ and $53 \mathrm{~m}$ respectively. Irregular waves in $\mathrm{S} 2$ were observed to break as far offshore as $x=42 \mathrm{~m}$. In MK1 and MK2 irregular waves start to break approximately at $h_{b}=12.5 \mathrm{~cm}$. In $\mathrm{S} 1$ and $\mathrm{S} 2$, the top of the bar is located between $x=52.8 \mathrm{~m}$ and $x=56.5 \mathrm{~m}$. In each case, $H_{0}$ is the farthest offshore measured wave height. 

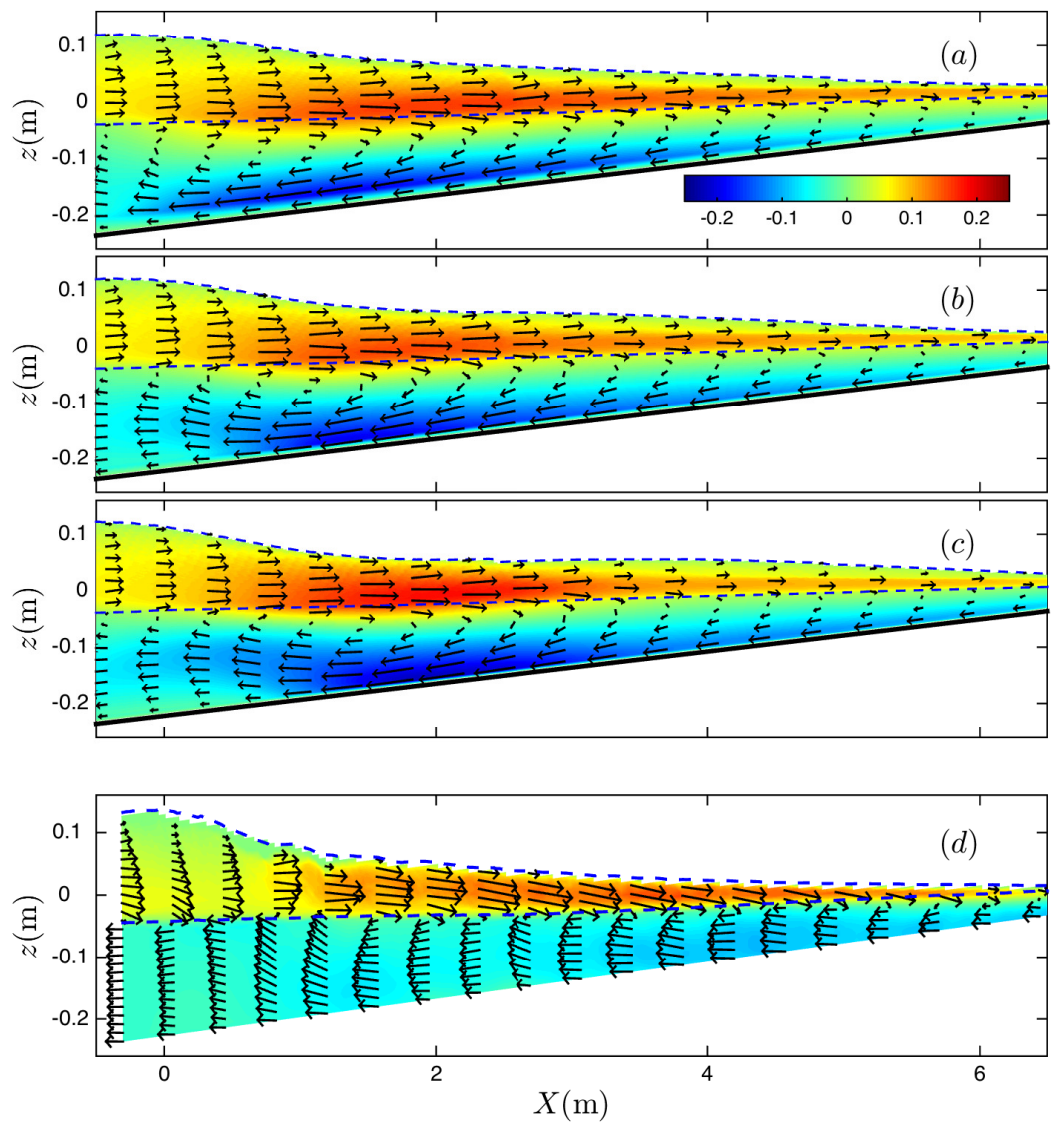

Figure 2: Time-averaged velocity field, $\overline{\mathbf{u}}$, for the regular depth-limited spilling breaking case TK1. Comparison between NHWAVE results with (a) $4 \sigma$-levels, (b) $8 \sigma$-levels, and (c) $16 \sigma$-levels as well as $(d)$ the 3-D VOF/LES simulation of Derakhti (2016). Dash lines show the crest $\langle\eta\rangle_{\max }$ and trough $\langle\eta\rangle_{\min }$ elevations. Colors show $\bar{u} / \sqrt{g \bar{D}} . X=x-x_{b}$ is the horizontal distance from the initial break point. 

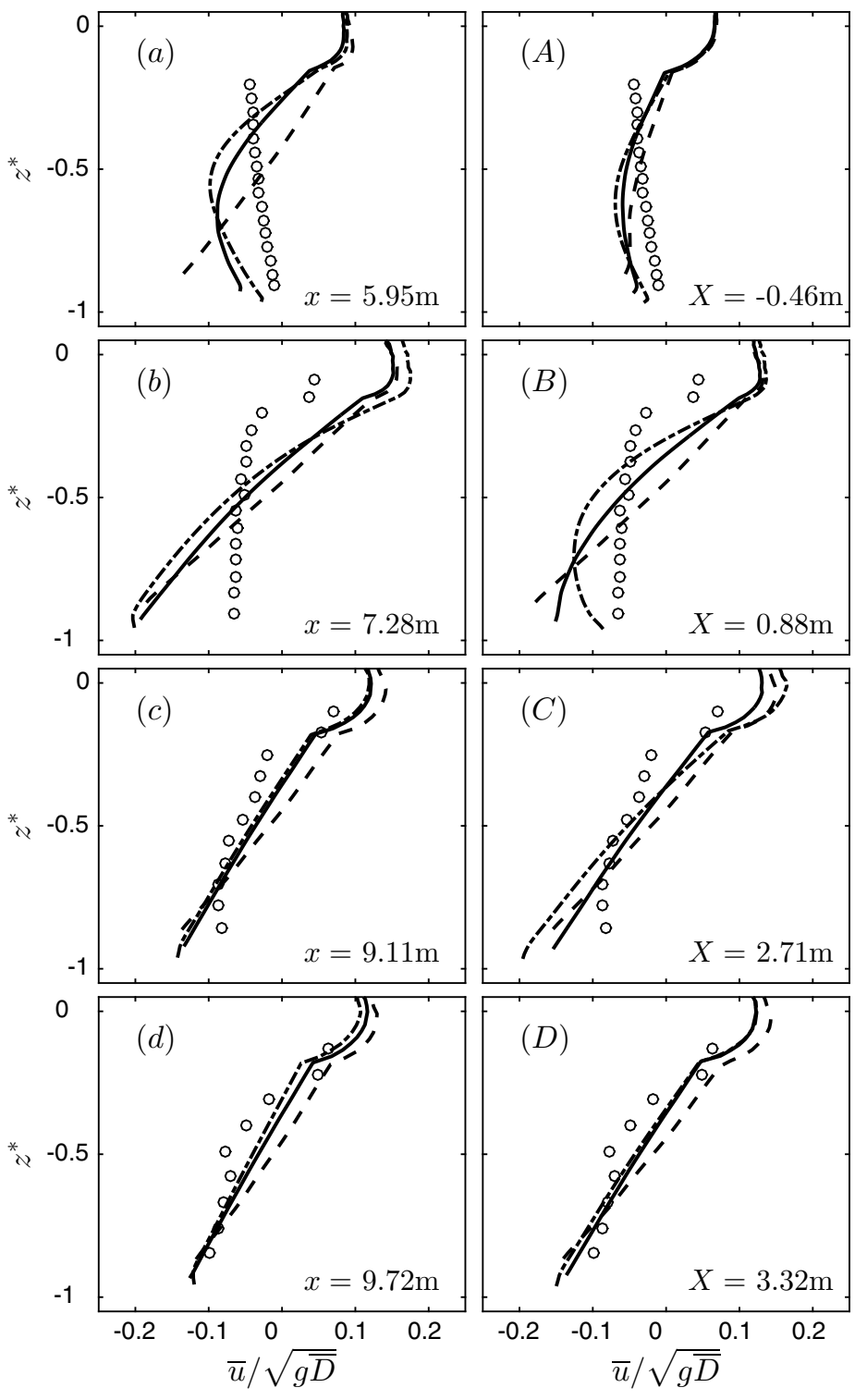

Figure 3: Time-averaged normalized horizontal velocity (undertow) profiles for the regular depth-limited spilling breaking case TK1 at $(a-d)$ different cross-shore locations, and $(A-D)$ different cross-shore locations in which the $x$ location of the predicted and observed results are shifted with respect to the simulated and observed break point, where the former is $0.7 \mathrm{~m}$ far more offshore than the latter in both cases. Here, $X=x-x_{b}$ represents the horizontal distance from the break point. Comparison between NHWAVE results with 4 $\sigma$-levels (dashed lines), $8 \sigma$-levels (solid lines), $16 \sigma$-levels (dotted-dashed lines) and the measurements (circle markers). Here, $z^{*}=(z-\bar{\eta}) / \bar{D}$ where $\bar{D}=h+\bar{\eta}$ is the mean water depth. 

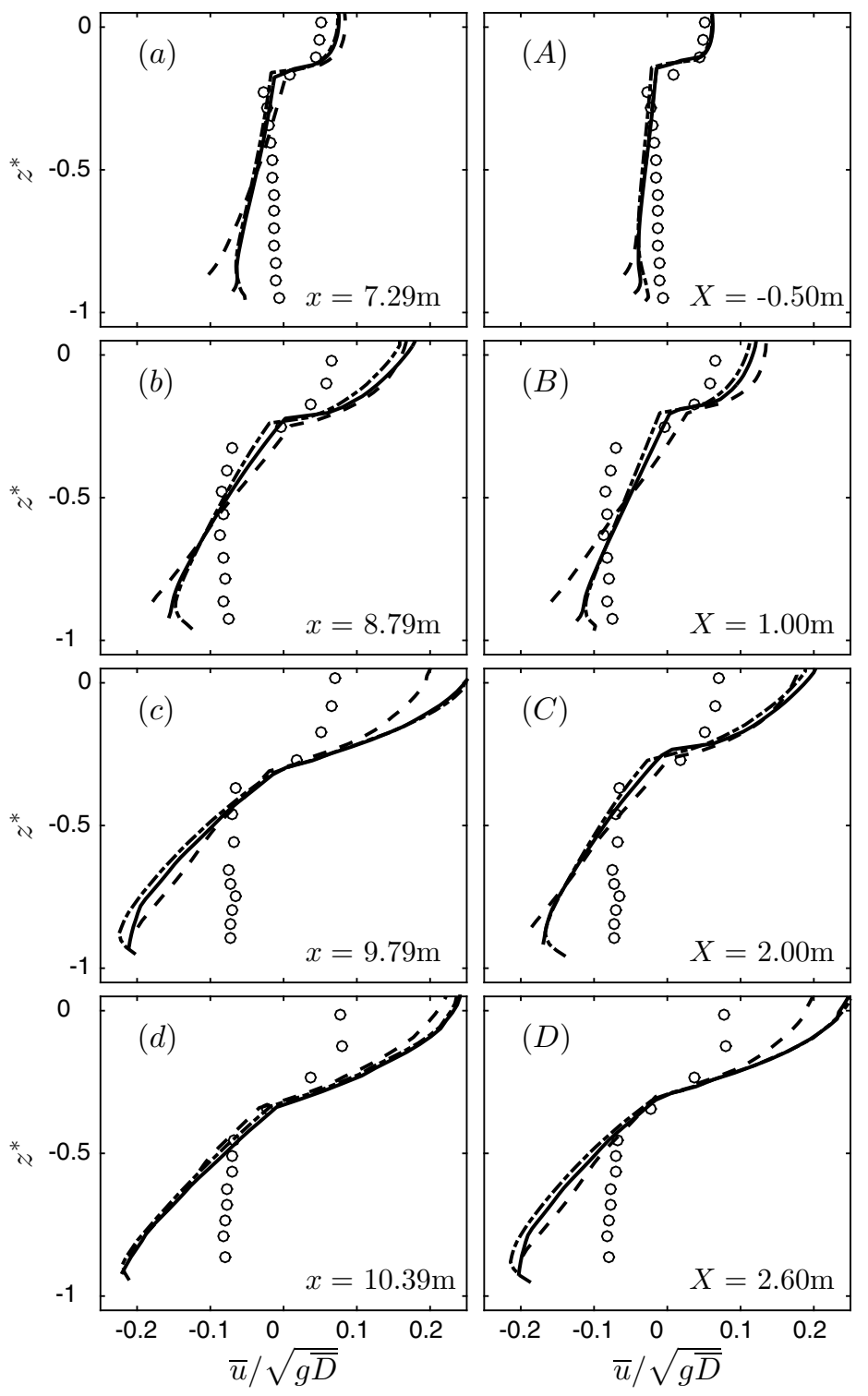

Figure 4: Time-averaged normalized horizontal velocity (undertow) profiles for the regular depth-limited plunging breaking case TK2 at $(a-d)$ different cross-shore locations, and $(A-D)$ different cross-shore locations in which the $x$ location of the predicted and observed results are shifted with respect to the simulated and observed break point, where the former is $0.7 \mathrm{~m}$ far more offshore than the latter in both cases. Here, $X=x-x_{b}$ represents the horizontal distance from the break point. Comparison between NHWAVE results with 4 $\sigma$-levels (dashed lines), $8 \sigma$-levels (solid lines), $16 \sigma$-levels (dotted-dashed lines) and the measurements (circle markers). Here, $z^{*}=(z-\bar{\eta}) / \bar{D}$ where $\bar{D}=h+\bar{\eta}$ is the mean water depth. 

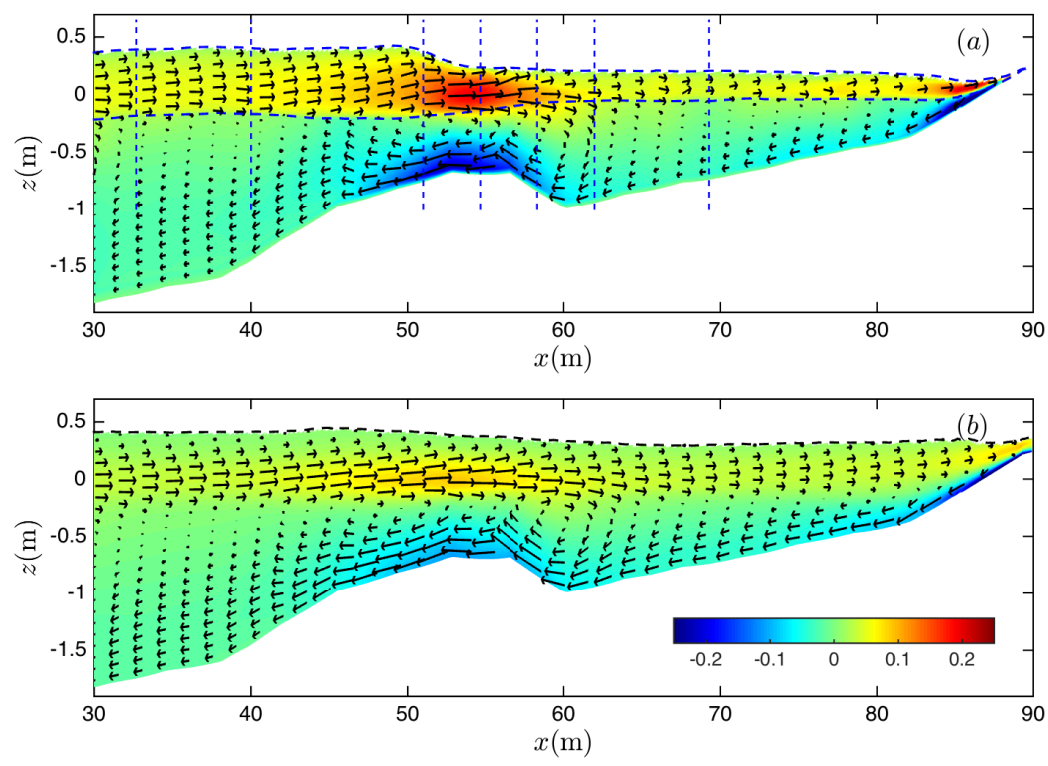

Figure 5: Time-averaged velocity field, $\overline{\mathbf{u}}$, for the $(a)$ regular S1 and (b) irregular S2 depthlimited breaking waves on a barred beach using $8 \sigma$-levels. In $(a)$, dash lines show the crest $\langle\eta\rangle_{\max }$ and trough $\langle\eta\rangle_{\min }$ elevations. In $(b)$, the dash line shows $H_{r m s}+\bar{\eta}$. Colors show $\bar{u} / \sqrt{g \bar{D}}$ where $\bar{D}=h+\bar{\eta}$ is the mean water depth. Vertical dashed lines show the cross-shore locations of the results shown in Figure 6 . The regular waves were observed to plunge at $x=53 \mathrm{~m}$. The random waves were observed to be both plunging and spilling as far offshore as $x=42 \mathrm{~m}$. 


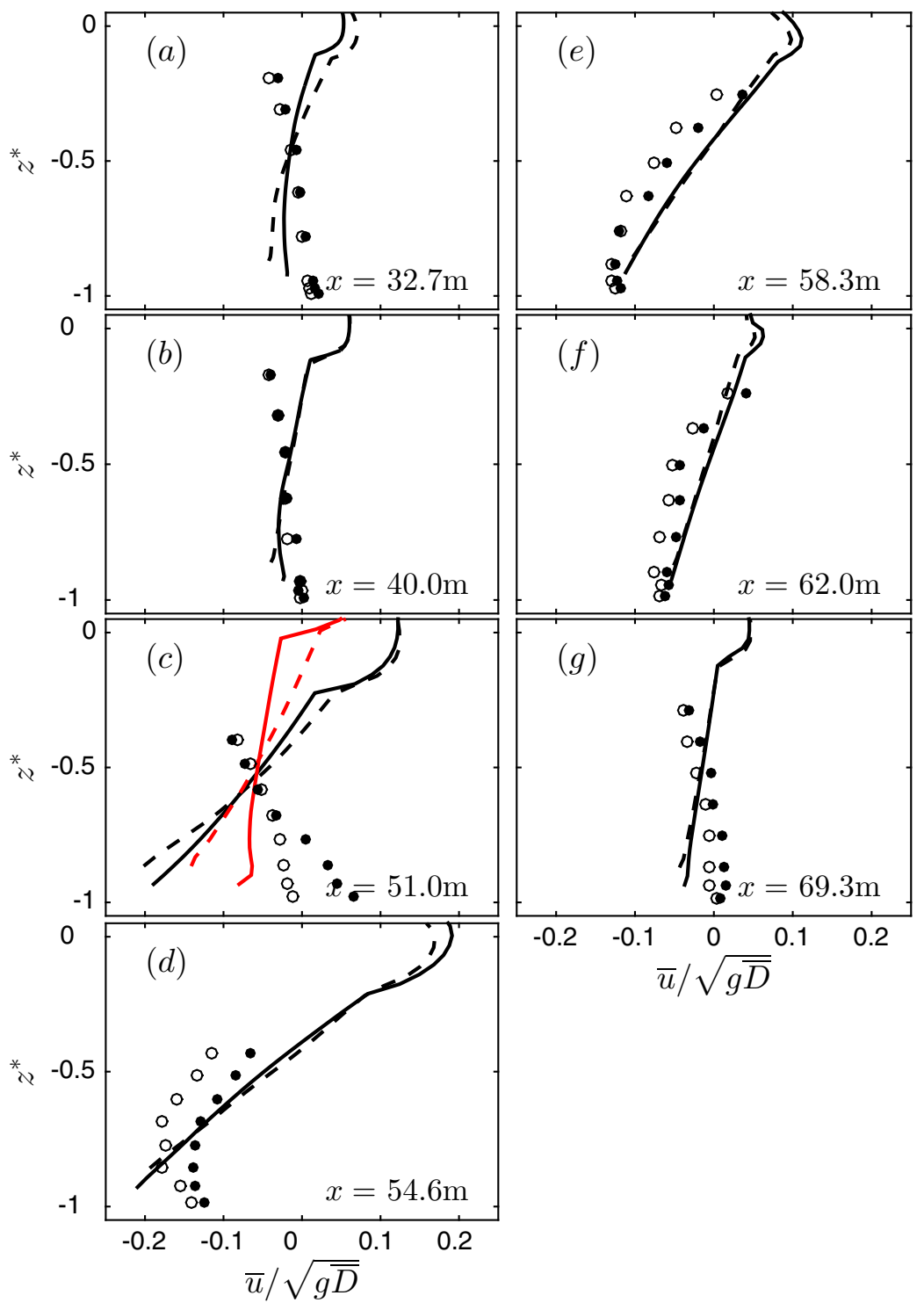

Figure 6: Time-averaged normalized horizontal velocity (undertow) profiles for the regular depth-limited plunging breaking waves on a barred beach case S1 at $(a-g)$ different crossshore locations before and after the bar. Comparison between NHWAVE results with 4 $\sigma$-levels (dashed lines), $8 \sigma$-levels (solid lines), and the measurements at two different longshore locations (open and solid circle markers). Red lines at $(c)$ show the results $3 \mathrm{~m}$ seaward of the corresponding measurement location. The regular waves were observed to plunge at $x=53 \mathrm{~m}$. Here, $\bar{D}=h+\bar{\eta}$ is the mean water depth. 


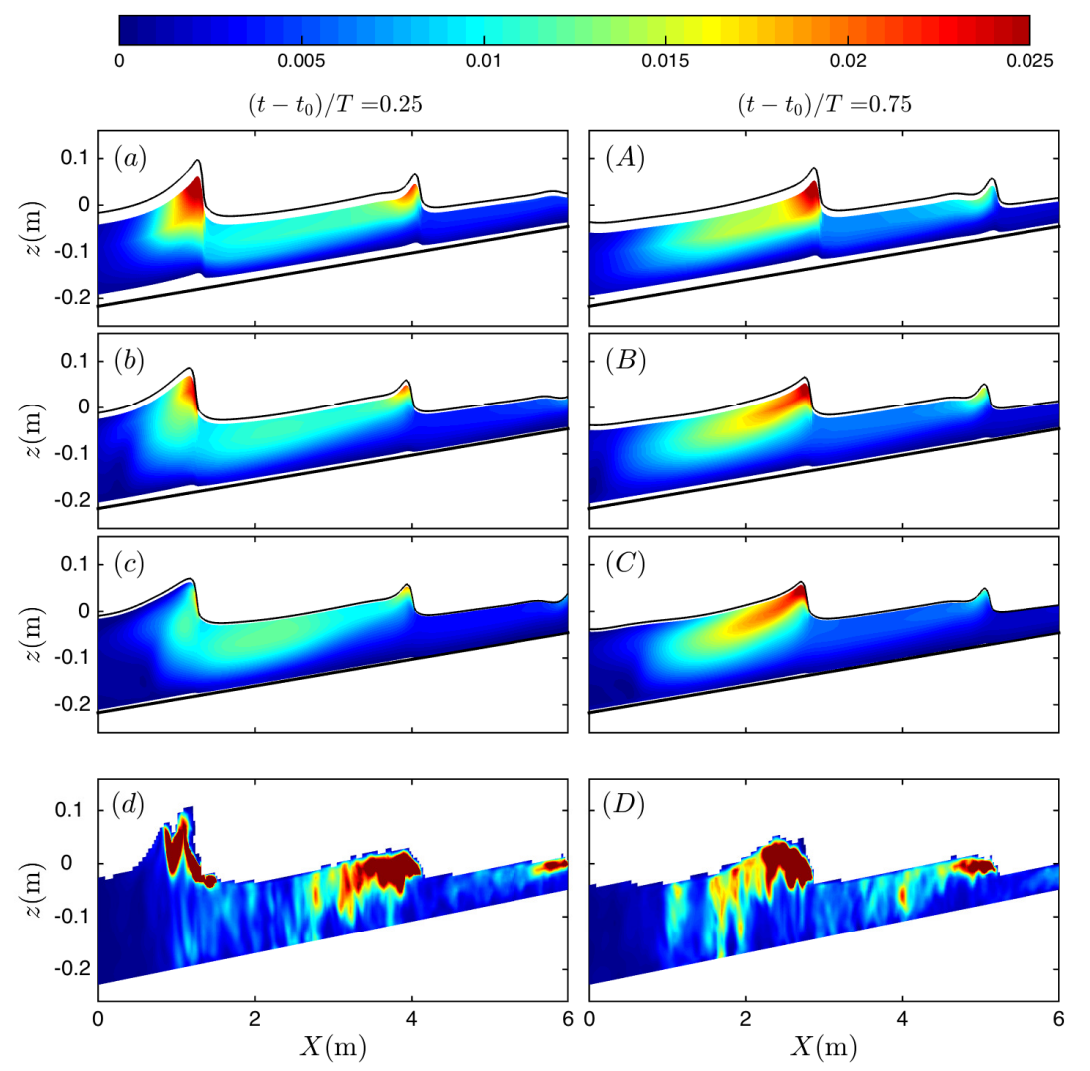

Figure 7: Snapshots of the turbulent kinetic energy, $k\left(\mathrm{~m}^{2} / \mathrm{s}^{2}\right)$, distribution at $(a-d)$ $\left(t-t_{0}\right) / T=0.25$ and $(A-D)\left(t-t_{0}\right) / T=0.75$ for the regular depth-limited spilling breaking case TK1. Comparison between NHWAVE results with $(a, A) 4 \sigma$-levels, $(b, B) 8$ $\sigma$-levels, and $(c, C) 16 \sigma$-levels as well as $(d, D)$ the 3 -D VOF/LES simulation of Derakhti (2016). Here, $X=x-x_{b}$ is the horizontal distance from the initial break point. 

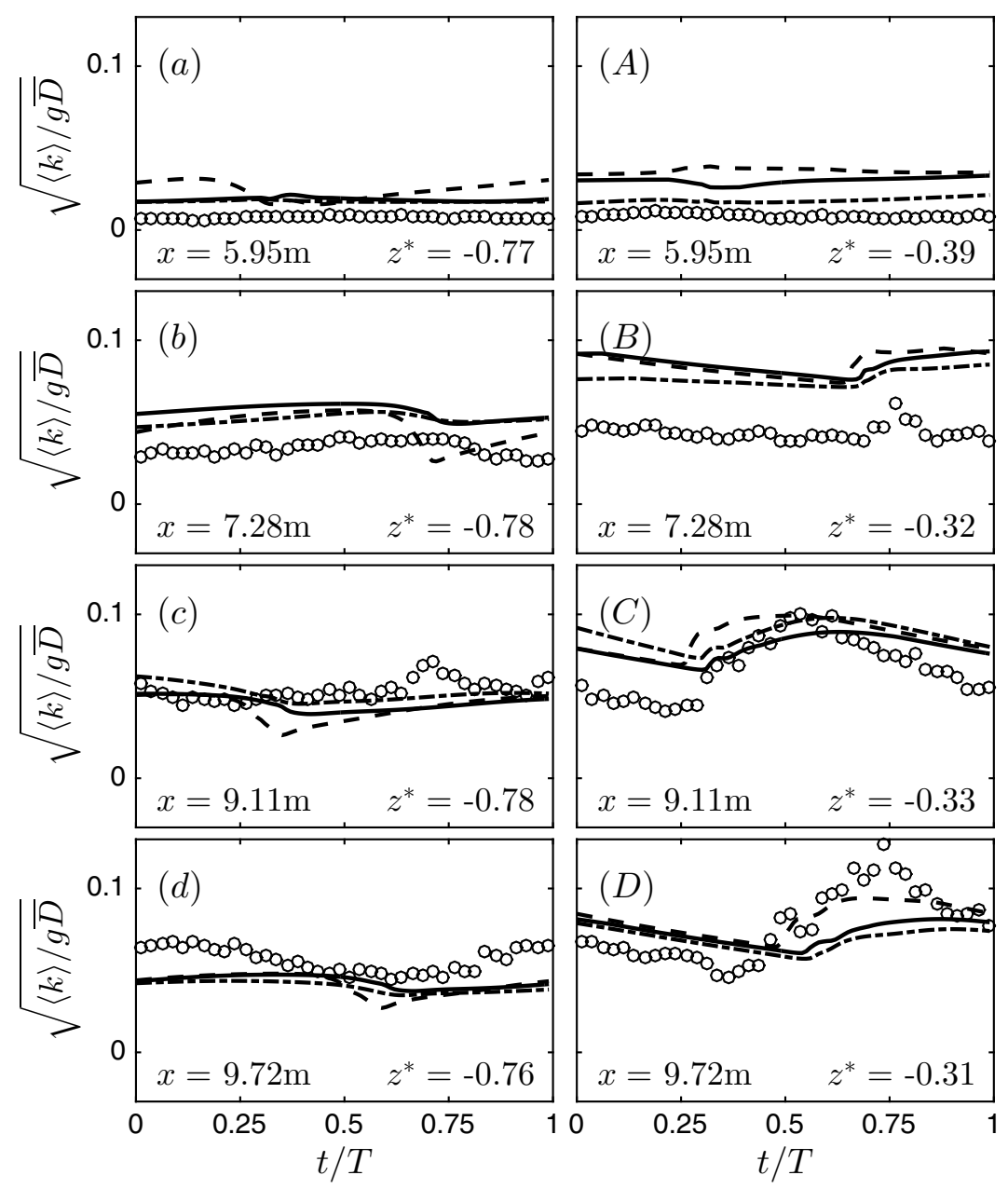

Figure 8: Phase-averaged $k$ time series for the regular depth-limited spilling breaking case TK1 close to the $(a-d)$ bed and $(A-D)$ trough level, at different cross-shore locations before and after the initial break point. Comparison between NHWAVE results with $4 \sigma$-levels (dashed lines), $8 \sigma$-levels (solid lines), $16 \sigma$-levels (dotted-dashed lines) and measurements (circle markers). Here, $z^{*}=(z-\bar{\eta}) / \bar{D}$ where $\bar{D}=h+\bar{\eta}$ is the mean water depth. The observed and simulated initial break points are at $x_{b}=6.4 \mathrm{~m}$ and $5.7 \mathrm{~m}$ respectively. 

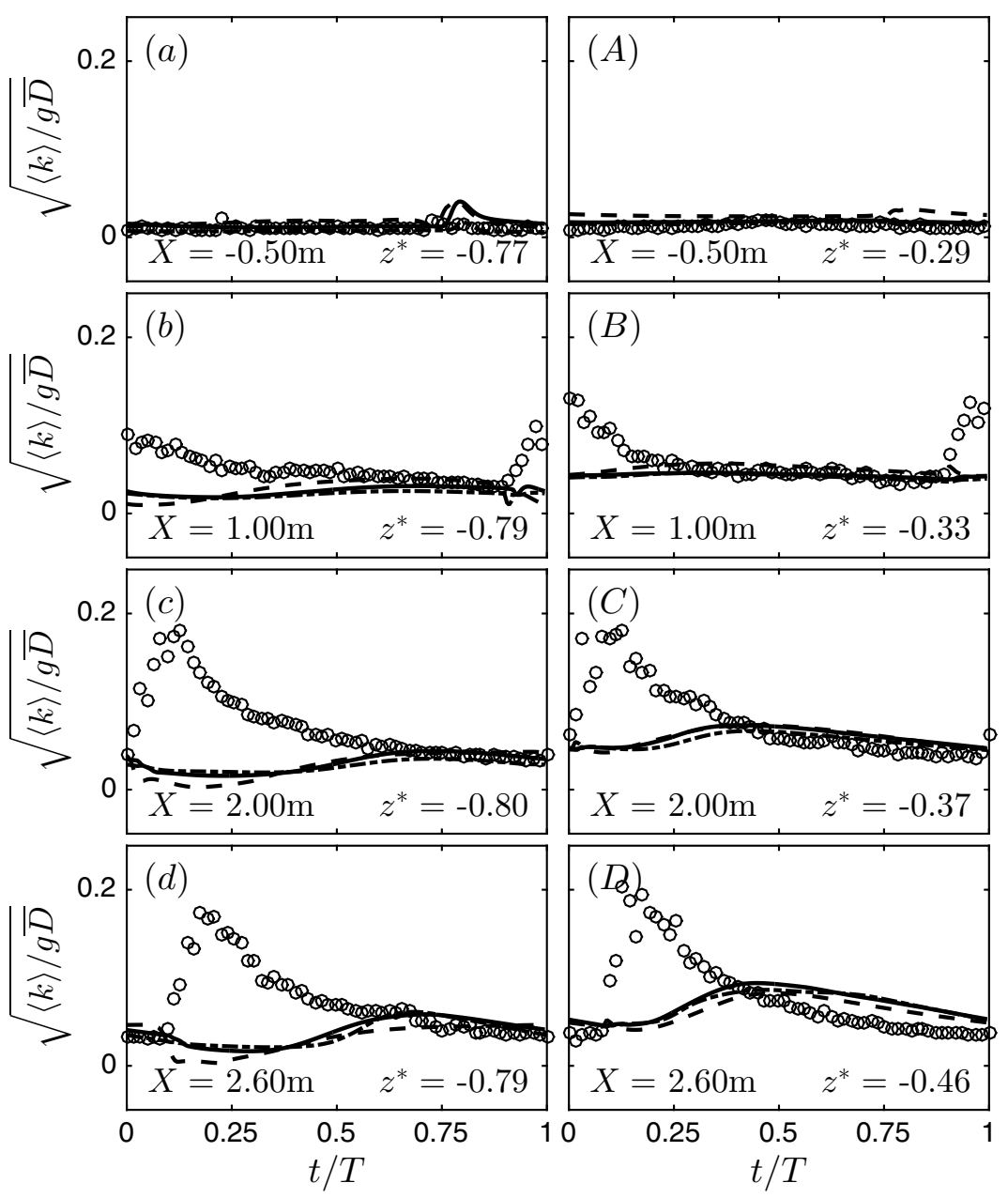

Figure 9: Phase-averaged $k$ time series for the regular depth-limited plunging breaking case TK2 close to the $(a-d)$ bed and $(A-D)$ trough level, at different cross-shore locations before and after the initial break point. Comparison between NHWAVE results with $4 \sigma$-levels (dashed lines), $8 \sigma$-levels (solid lines), $16 \sigma$-levels (dotted-dashed lines) and measurements (circle markers). Here, $z^{*}=(z-\bar{\eta}) / \bar{D}$ where $\bar{D}=h+\bar{\eta}$ is the mean water depth, and $X=x-x_{b}$ is the horizontal distance from the initial break point, where the observed and simulated initial break points are at $x_{b}=7.8 \mathrm{~m}$ and $7.1 \mathrm{~m}$ respectively. 

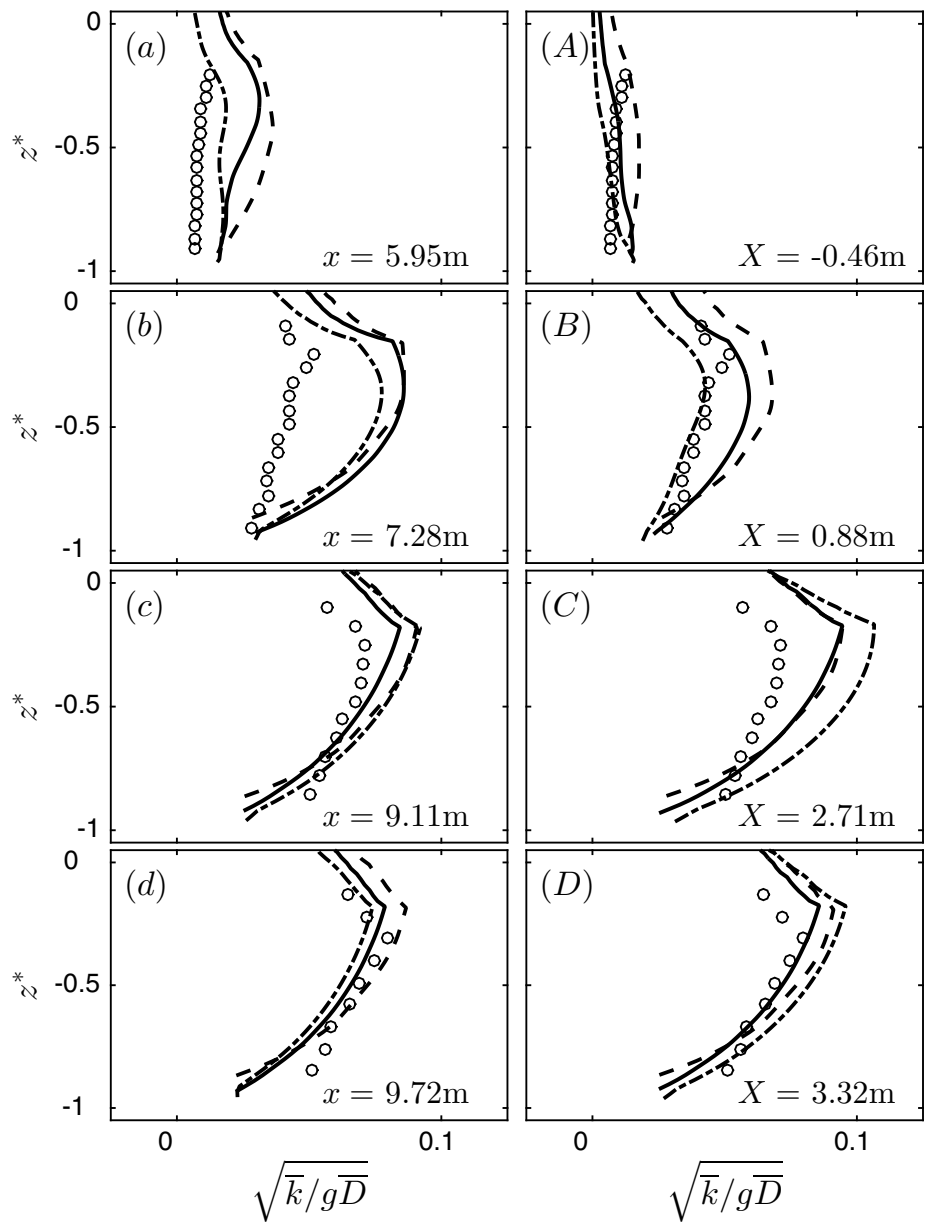

Figure 10: Time-averaged normalized $k$ profiles for the regular depth-limited spilling breaking case TK1 at at $(a-d)$ different cross-shore locations, and $(A-D)$ different cross-shore locations in which the $x$ location of the predicted and observed results are shifted with respect to the simulated and observed break point, where the former is $0.7 \mathrm{~m}$ far more offshore than the latter in both cases. Here, $X=x-x_{b}$ represents the horizontal distance from the break point, and $z^{*}=(z-\bar{\eta}) / \bar{D}$ where $\bar{D}=h+\bar{\eta}$ is the mean water depth. Comparison between NHWAVE results with $4 \sigma$-levels (dashed lines), $8 \sigma$-levels (solid lines), $16 \sigma$-levels (dotted-dashed lines) and the measurements (circle markers). 

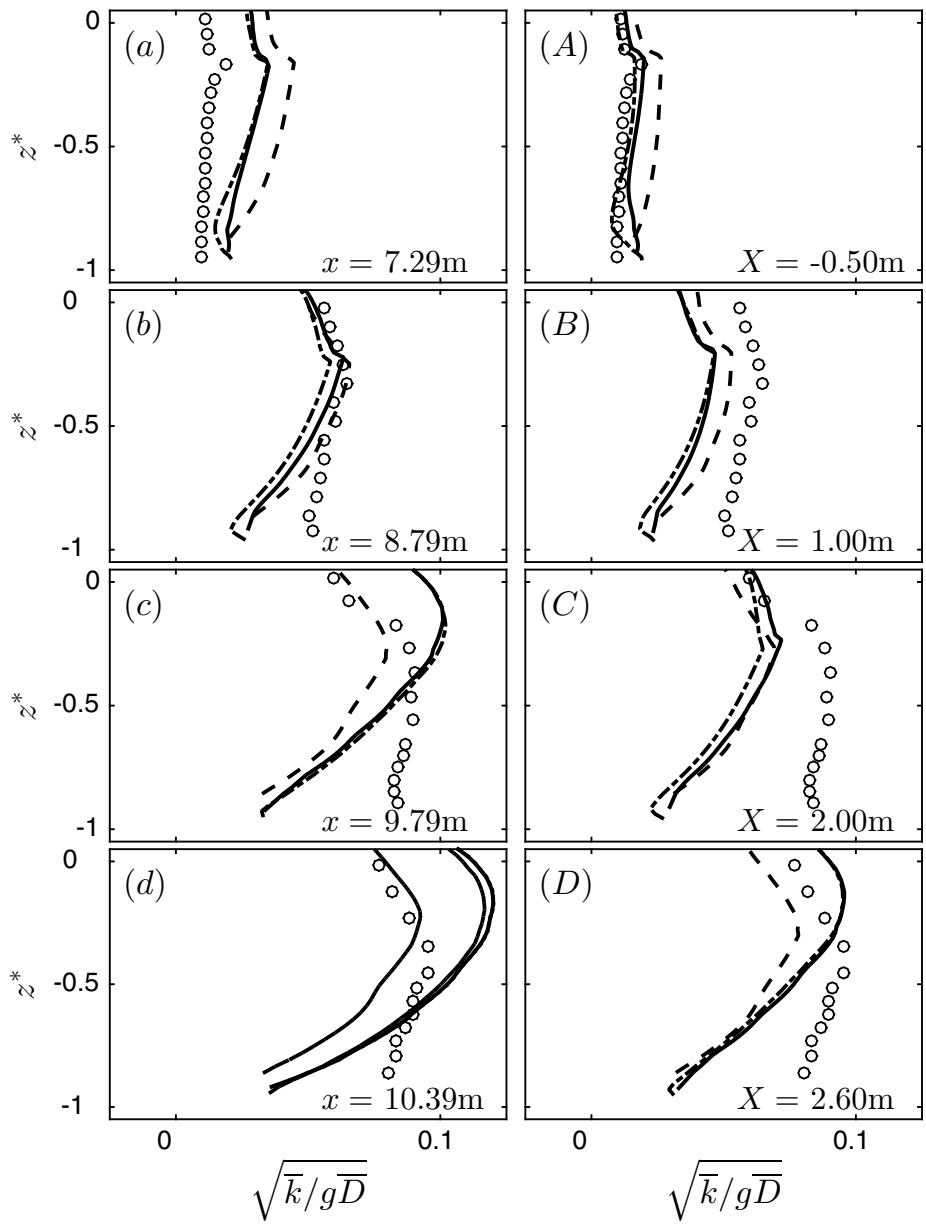

Figure 11: Time-averaged normalized $k$ profiles for the regular depth-limited plunging breaking case TK2 at $(a-d)$ different cross-shore locations, and $(A-D)$ different crossshore locations in which the $x$ location of the predicted and observed results are shifted with respect to the simulated and observed break point, where the former is $0.7 \mathrm{~m}$ far more offshore than the latter in both cases. Here, $X=x-x_{b}$ represents the horizontal distance from the break point, and $z^{*}=(z-\bar{\eta}) / \bar{D}$ where $\bar{D}=h+\bar{\eta}$ is the mean water depth. Comparison between NHWAVE results with $4 \sigma$-levels (dashed lines), $8 \sigma$-levels (solid lines), $16 \sigma$-levels (dotted-dashed lines) and the measurements (circle markers). 

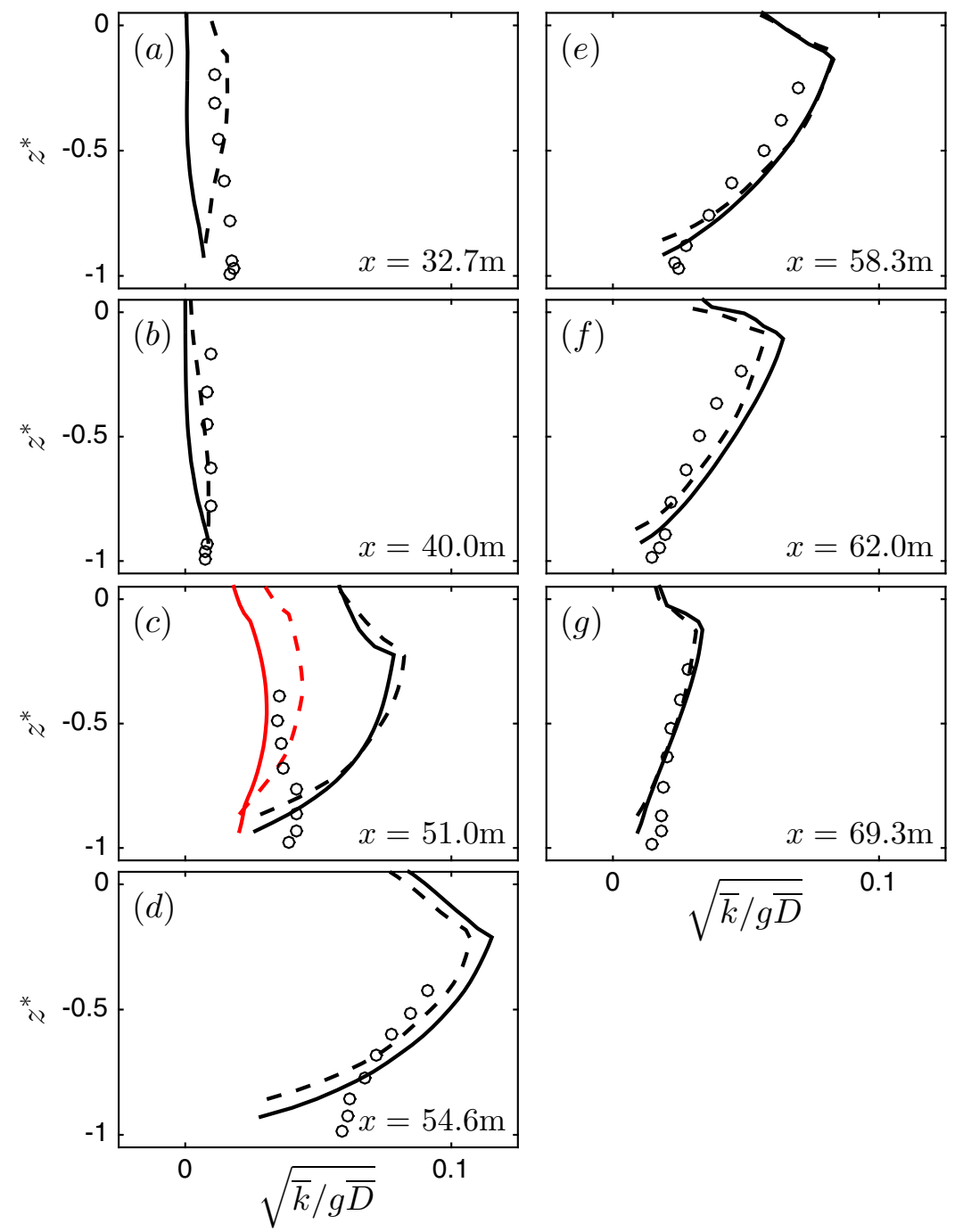

Figure 12: Time-averaged normalized $k$ profiles for the surf zone regular breaking waves on a barred beach case S1 at different cross-shore locations before and after the bar. Comparison between NHWAVE results with $4 \sigma$-levels (dashed lines), $8 \sigma$-levels (solid lines), and the measurements (circle markers). Red lines at $(c)$ show the results $3 \mathrm{~m}$ seaward of the corresponding measurement location. Here, $z^{*}=(z-\bar{\eta}) / \bar{D}$ where $\bar{D}=h+\bar{\eta}$ is the mean water depth. 

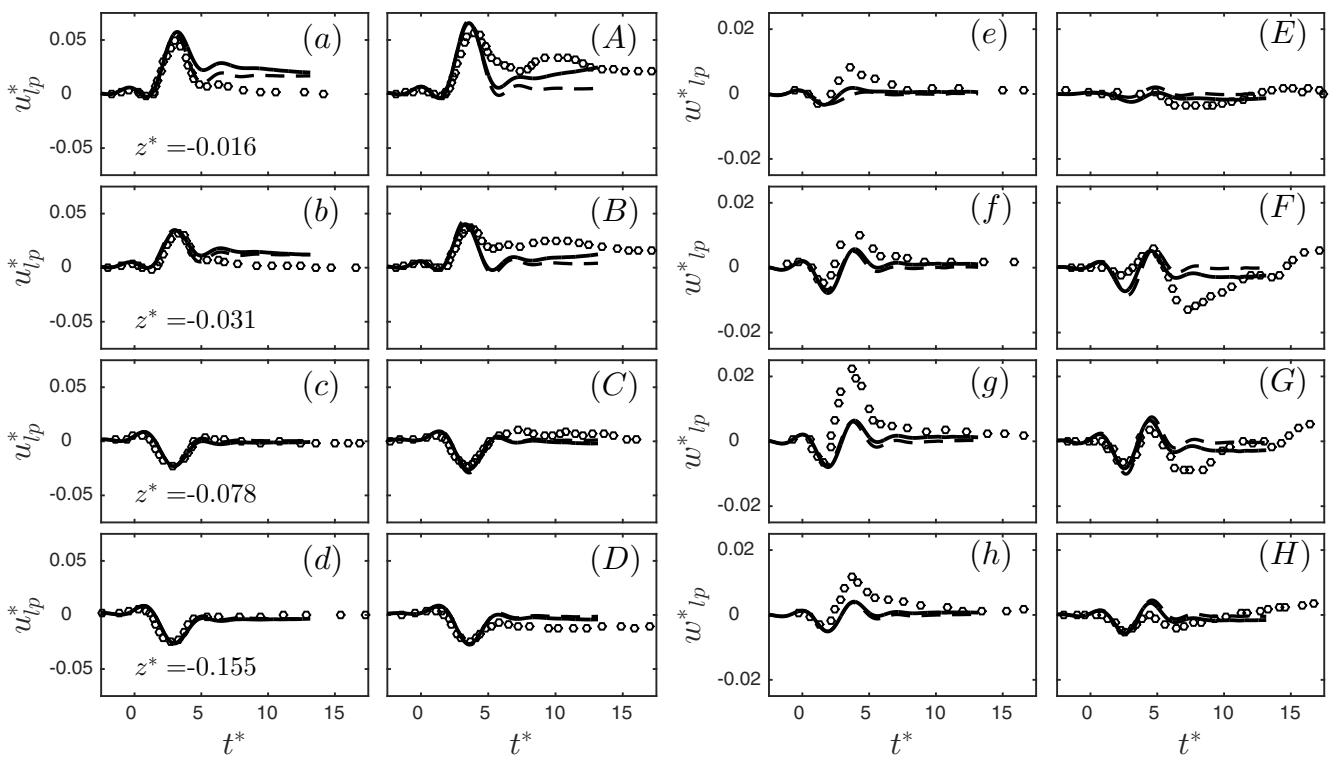

Figure 13: Normalized low-pass filtered horizontal and vertical velocities for RM1 using $8 \sigma$-levels (dashed lines) and $16 \sigma$-levels (solid lines), at $(a-h) x^{*}=0.15$ and $(A-$ $H) x^{*}=0.60$ at different elevations $(a, A, e, E) z^{*}=-0.016,(b, B, f, F) z^{*}=-0.031$, $(c, C, g, G) z^{*}=-0.078,(d, D, h, H) z^{*}=-0.155$. The circles are the measurements of the corresponding case adopted from Rapp \& Melville (1990), Figure 42. The reference value is the wave phase speed of the center frequency wave component of the input packet. 

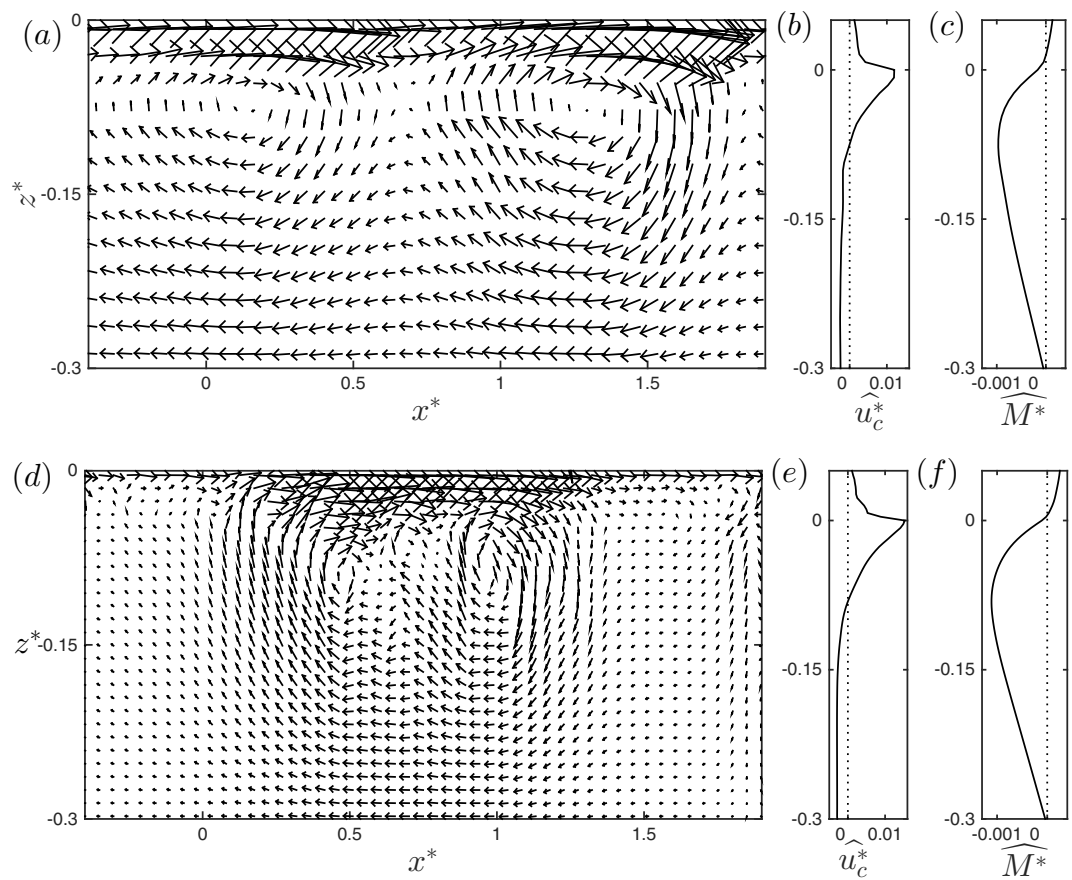

Figure 14: $(a, d)$ Spatial distribution of the normalized mean current, $\mathbf{u}_{\mathbf{c}}^{*} ;(b, e)$ normalized horizontal-averaged mean current in the streamwise direction, $\widehat{u_{c}^{*}}$ and $(c, f)$ normalized accumulative horizontal-averaged mass flux, $\widehat{M^{*}}$, in the breaking region for RM1. $(a-c)$ NHWAVE results with $8 \sigma$-levels and $(d-f)$ VOF/LES results by Derakhti \& Kirby $(2014 a)$. 

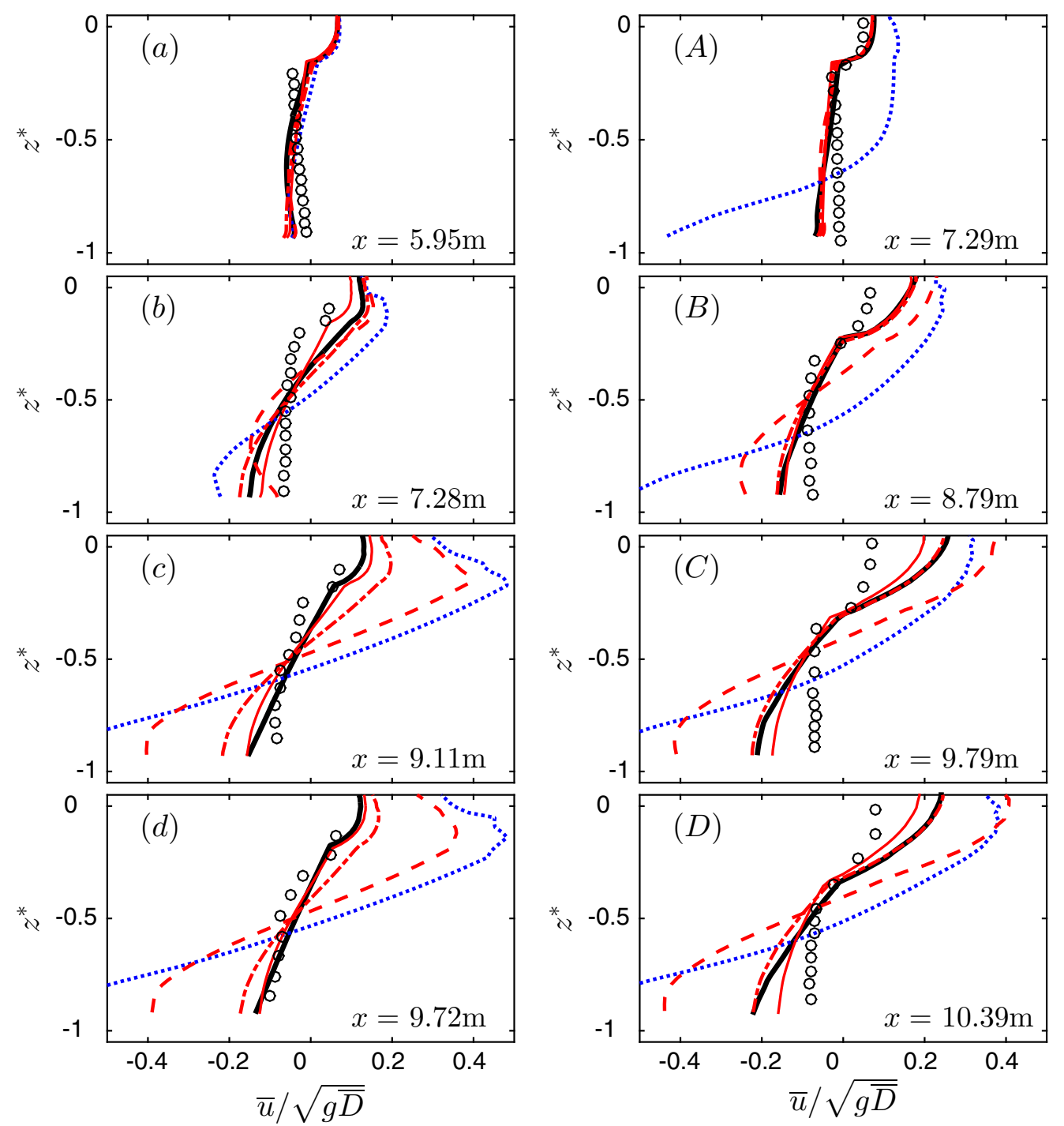

Figure 15: Time-averaged normalized horizontal velocity (undertow) profiles for the regular depth-limited $(a-d)$ spilling TK1 and $(A-D)$ plunging TK2 breaking cases at different cross-shore locations before and after the initial break point. Comparison between the NHWAVE results in which $\nu_{t}$ is calculated based on the $k-\epsilon$ turbulence model (black thick solid lines), $\nu_{t}=0$ (blue dotted lines), $\nu_{t} / \nu=100$ (red dashed lines), $\nu_{t} / \nu=500$ (red dotted-dashed lines), and $\nu_{t} / \nu=1000$ (red thin solid lines) as well as and the measurements of Ting \& Kirby (1994) (markers). The observed break point for TK1 and TK2 are at $x_{b}=6.4 \mathrm{~m}$ and $7.8 \mathrm{~m}$, respectively. 


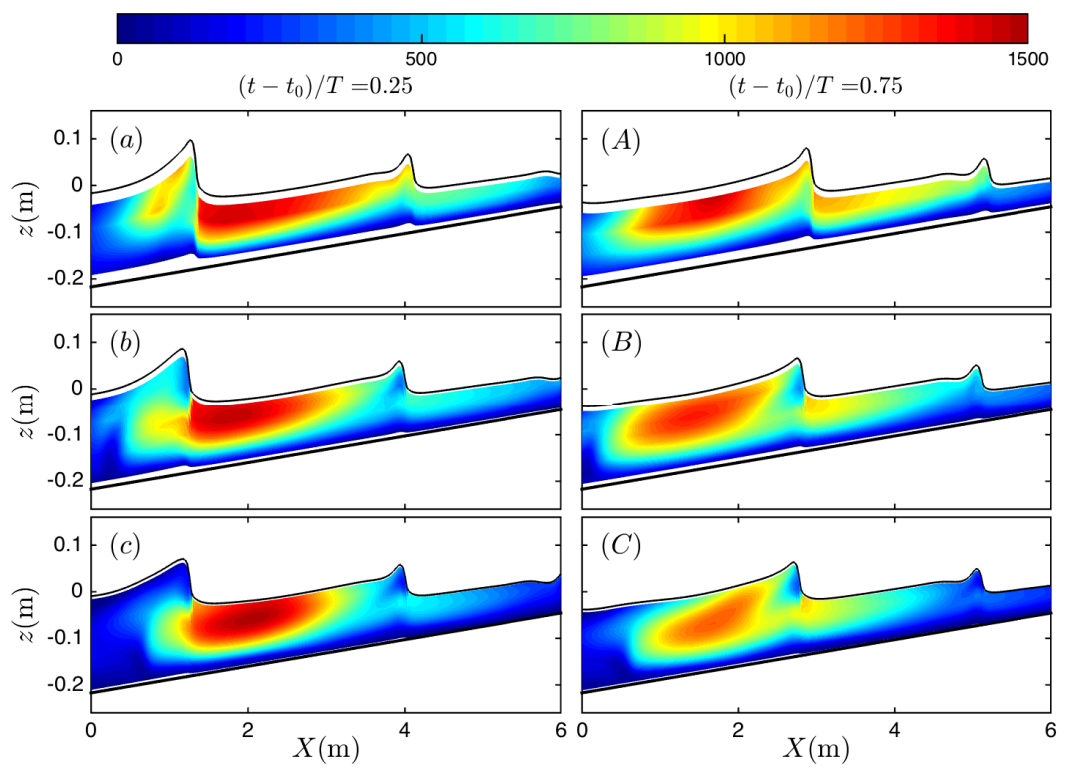

Figure 16: Snapshots of the normalized turbulent eddy viscosity, $\nu_{t} / \nu$, distribution at $(a-c)\left(t-t_{0}\right) / T=0.25$ and $(A-C)\left(t-t_{0}\right) / T=0.75$ for the regular depth-limited spilling breaking case TK1 using $(a, A) 4 \sigma$-levels, $(b, B) 8 \sigma$-levels and $(c, C) 16 \sigma$-levels. Here, $X=x-x_{b}$ is the horizontal distance from the initial break point. 